\title{
Concatenated Catalytic Asymmetric Allene Diboration/Allylation/Functionalization
}

Angela R. Woodward, Heather E. Burks, Louis M. Chan and James P. Morken*

\section{Supporting Information}

General. ${ }^{1} \mathrm{H}$ NMR spectra were recorded on Bruker DRX 300, 400, or $500 \mathrm{MHz}$ spectrometers. Chemical shifts are reported in ppm from tetramethylsilane with the solvent resonance as the internal standard $\left(\mathrm{CDCl}_{3}: 7.26 \mathrm{ppm}\right)$. Data are reported as follows: chemical shift, integration, multiplicity $(\mathrm{s}=$ singlet, $\mathrm{d}=$ doublet, $\mathrm{t}=$ triplet, $\mathrm{q}=$ quartet, $\mathrm{br}=$ broad, $\mathrm{m}=$ multiplet $)$ and coupling constants $(\mathrm{Hz}) .{ }^{13} \mathrm{C}$ NMR was recorded on a Bruker $400 \mathrm{MHz}(100 \mathrm{MHz})$ or $500 \mathrm{MHz}(125 \mathrm{MHz})$ spectrometer with complete proton decoupling. ${ }^{31} \mathrm{P}$ NMR spectra were recorded on a Bruker $400 \mathrm{MHz}(161 \mathrm{MHz})$ spectrometer with complete proton decoupling. Chemical shifts are reported in ppm from tetramethylsilane with the solvent as the internal standard $\left(\mathrm{CDCl}_{3}: 77.0 \mathrm{ppm}\right)$. High-resolution and low resolution mass spectrometry were performed by the University of Minnesota or the University of North Carolina at Chapel Hill Mass Spectrometry Service Laboratory. Infrared (IR) spectra were obtained on an ASI ReactIR 1000. Bands are characterized as broad (br), strong (s), and medium (m).

Liquid chromatography was performed using forced flow (flash chromatography) on silica gel ( $\mathrm{SiO}_{2}, 230$ X $450 \mathrm{mesh}$ ) purchased from EMD Chemicals, Inc. Thin layer chromatography was performed on 250 $\mu \mathrm{m}$ silica gel plates from Sorbent Technologies, Inc. Visualization was achieved using UV light, phosphomolybdic acid in ethanol, potassium permanganate in water, or cerium sulfate and ammonium molybdate in sulfuric acid, each followed by heating.

Analytical gas-liquid chromatography (GLC) was performed on a Hewlett-Packard 6890 Series chromatography equipped with a CTC Analysis Combi Pal autosampler by Leap Technologies (Carrboro, NC), a split mode capillary injection system, a flame ionization detector and a Supelco $\beta$-dex 120 column with helium as the carrier gas. Analytical supercritical fluid chromatography (SFC) was performed on a Berger Instruments supercritical chromatograph equipped with an Alcott autosampler and a Knauer UV detector.

Diboration/allylation reactions were conducted in oven and flame dried glassware under an inert atmosphere of nitrogen or argon. Toluene was distilled over $\mathrm{CaH}_{2}$ and degassed by freeze-pump-thaw cycles prior to use. Tris(dibenzylideneacetone)dipalladium was purchased from Strem Chemical Company. Allenes were synthesized according to the literature [(a) Vermeer, P.; Meijer, J.; Brandsma, L. Recl. Trav. Chim. Pays-Bas 1975, 94, 112 (b) Myers, A. G.; Zheng, B. J. Am. Chem. Soc. 1996, 118, 4492]. Racemic compounds were prepared by the diboration of allenes using $\mathrm{PCy}_{3}$ as the ligand. Isobutyraldehyde, butyraldehyde, benzaldehyde and iodobenzene were freshly distilled prior to use. All other reagents were purchased from Strem, Aldrich, BASF, Cambridge Isotopes, Matrix, or Alfa Aesar Chemical Companies and used directly. Benzoate derivatives of the $\beta$-hydroxyketone products for SFC analysis were prepared by treatment of the $\beta$-hydroxyketones with benzoyl chloride, pyridine, and 4dimethylaminopyridine in $\mathrm{CH}_{2} \mathrm{Cl}_{2}$ followed by silica gel chromatography. 


\section{General Procedure for Monitoring Conversion for the Formation of 1,2-Bis(boronate)esters}

To a two-dram oven dried vial equipped with magnetic stir was added tris(dibenzylideneacetone)dipalladium(0) $(3.47 \mathrm{mg}, 0.0038 \mathrm{mmol})$ and the respective TADDOLphosphoramidite $(0.00912 \mathrm{mmol})$ in $300 \mu \mathrm{L}$ toluene- $d_{8}$ in an inert atmosphere dry box. This mixture was stirred for $60 \mathrm{~min}$ at which time bis(pinacolato)diboron (46.3 $\mathrm{mg}, 0.1824 \mathrm{mmol}, 1.2 \mathrm{equiv}$ ) and the respective allene $(0.152 \mathrm{mmol})$ were added. The mixture was transferred to an oven dried J-Young Tube, an additional $700 \mu \mathrm{L}$ of toluene- $d_{8}$ was added, and the reaction was removed from the dry box. After 12 or $24 \mathrm{~h}$, the reaction was charged with trimethoxybenzene and the reaction was monitored by ${ }^{1} \mathrm{H}$ NMR and the amount of allene consumed was calculated. The isolated yields for 1,2-bis(boronate) esters were determined from reactions using this general procedure but without the addition of internal standard. The allene diboration adducts have been previously characterized (Pelz et. al J. Am. Chem. Soc. 2004, 126, 16328).

\section{Preparation and Characterization of TADDOLPNMe $:$}

Alexakis, A.; Burton, J.; Vastra, J.; Benhaim, C.; Fournioux, X.; Heuvel, A. V. D.; Levêque, J. M.; Mazé, F.; Rosset, S. Eur. J. Org. Chem. 2000, 4011.

\section{Preparation and Characterization of 3,5-Me $\mathrm{MADDOLPNMe}_{2}$ :}

Boele, M.D.K.; Kamer, P.C.J.; Lutz, M.; Spek, A.L.; de Vries, J.G.; van Leeuwen, P.W. N. M.; vanStrijdonck, G.P.F. Chem. Eur. J. 2004, 10, 6232.

\section{Procedure for the Preparation of 3,5-( $\left.{ }^{t} \mathrm{Bu}\right)_{2}$ TADDOLPNMe ${ }_{2}$ :}

To a $250-\mathrm{mL}$ flame-dried round bottom flask equipped with an oven dried magnetic stir bar was added $4 \AA$ molecular sieves, followed by 3,5-( $\left.{ }^{t} \mathrm{Bu}\right)_{2}$ TADDOL (prepared from L-tartaric acid and 1-bromo-3,5-ditert-butyl benzene, see: Seebach, D.; Beck, A.K.; Keckel, A. Angew. Chem. Int. Ed. 2001, 40, 92-138.) (5.573 g $6.087 \mathrm{mmol}, 1$ equiv) and tetrahydrofuran $(25 \mathrm{~mL}, 0.25 \mathrm{M})$. The flask was cooled to $0{ }^{\circ} \mathrm{C}$ (icewater bath) and charged with triethylamine $(2.88 \mathrm{~mL}, 20.69 \mathrm{mmol}, 3.4$ equiv) and phosphorus trichloride (637 $\mu \mathrm{L}, 7.305 \mathrm{mmol}, 1.2$ equiv). The mixture was allowed to warm to ambient temperature and was stirred for $30 \mathrm{~min}$, at which time it was cooled to $0{ }^{\circ} \mathrm{C}$ (ice-water bath) and charged with dimethyl amine (30 mL, 2.0 M in tetrahydrofuran, 10 equiv). The mixture was warmed to ambient temperature and was allowed to stir overnight before it was diluted with diethyl ether and filtered over Celite. The solvent was removed via rotary evaporation and the crude reaction mixture was purified on silica gel (4\% ethyl acetate/Hexanes) to afford the desired phosphoramidite (3.95 g, 65\% yield).

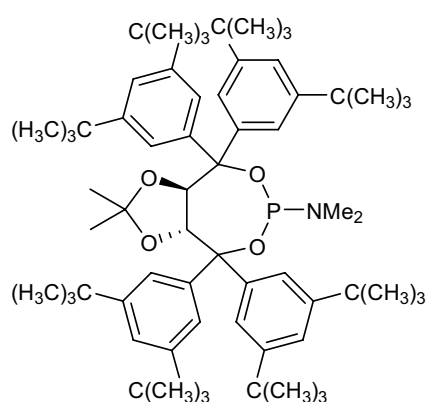

Page S- 2
Dimethyl-[4,4,8,8-tetrakis-(3,5-di-tert-buyl-phenyl)-2,2-dimethyl-tetrahydro-[1,3] dioxolo[4,5-e][1,3,2] dioxaphosphepin-6-yl]-amine. IR $\left(\mathrm{CH}_{2} \mathrm{Cl}_{2}\right)$ : 3437 (w, br), 3064 (s), 2959 (s), 2900 (s), 2854 (s), 2792 (s), 2753 (s), 2709 (s), 2353 (s), 2326 (s), 2287 (s), 1778 (s), 1592 (s), 1475 (s), 1392 (s), 1366 (s), 1254 (s), 1190 (s), 1053 (s), 968 (s), 894 (s), 870 (s), 785 (s), 734 (s), 696 (s). $\mathrm{cm}^{-1} ;{ }^{1} \mathrm{H}$ NMR $\left(400 \mathrm{MHz}, \mathrm{CDCl}_{3}\right): \delta 0.08\left(3 \mathrm{H}, \mathrm{s}, \mathrm{CH}_{3} \mathrm{CO}\right), 1.24(18 \mathrm{H}, \mathrm{s}$, $\left.\left(\mathrm{CH}_{3}\right)_{3} \mathrm{CAr}\right), 1.26\left(18 \mathrm{H}, \mathrm{s},\left(\mathrm{CH}_{3}\right)_{3} \mathrm{CAr}\right), 1.28\left(18 \mathrm{H}, \mathrm{s},\left(\mathrm{CH}_{3}\right)_{3} \mathrm{CAr}\right), 1.29(18 \mathrm{H}$, 
s, $\left.\left(\mathrm{CH}_{3}\right)_{3} \mathrm{CAr}\right), 1.46\left(3 \mathrm{H}, \mathrm{s}, \mathrm{CH}_{3} \mathrm{CO}\right), 2.88\left(6 \mathrm{H}, \mathrm{d}, J=10.4 \mathrm{~Hz},\left(\mathrm{CH}_{3}\right)_{2} \mathrm{~N}\right), 4.76(1 \mathrm{H}, \mathrm{d}, J=8.8 \mathrm{~Hz}$, OCHC), 5.25 (1H, dd, $J=8.4,2.4 \mathrm{~Hz}, \mathrm{OCHC}), 7.12$ (2H, d, $J=2 \mathrm{~Hz}, \mathbf{H}-\mathrm{Ar}), 7.20-7.25$ (4H, m, H-Ar), 7.41 (2H, s, H-Ar), $7.60(2 \mathrm{H}, \mathrm{d}, J=1.6 \mathrm{~Hz}, \mathbf{H}-\mathrm{Ar}), 7.63(2 \mathrm{H}, \mathrm{d}, J=1.6 \mathrm{~Hz}, \mathbf{H}-\mathrm{Ar}) .{ }^{13} \mathrm{C}$ NMR $(100 \mathrm{MHz}$, $\left.\mathrm{CDCl}_{3}\right): \delta 24.08,28.23,31.65,31.69,31.7,34.96,35.09,35.17,35.57,35.77,81.52,81.49,82.44,82.50$, $83.90,83.94,84.11$ (the region $81.52-84.11 \mathrm{ppm}$ contains extra carbon peaks, possibly due to C-P coupling, see: Boele, M.D.K.; Kamer, P.C.J.; Lutz, M.; Spek, A.L.; de Vries, J.G.; van Leeuwen, P.W.N.M.; van Strijdonck, G.P.F. Chem. Eur. J. 2004, 10, 6232), 110.03, 120.11, 120.40, 120.72, 121.62, $123.80,123.96,123.99,141.26,142.01,146.20,146.23,146.66,148.89,149.05,149.37,149.76 .{ }^{31} \mathrm{P}$ NMR $\left(161 \mathrm{MHz}, \mathrm{CDCl}_{3}\right): \delta$ 141.98. HRMS (ESI) Calc'd for $\mathrm{C}_{65} \mathrm{H}_{98} \mathrm{NO}_{4} \mathrm{P}(\mathrm{M}+\mathrm{H})^{+}: 988.7312$ Found $(\mathrm{M}+\mathrm{H})^{+}: 988.7306$.

\section{Determination of Enantiopurity of 1,2-Bis(boronate)esters}

Enantioselectivity was determined by diimide reduction (2-nitrobenzenesulfonylhydrazide, triethylamine, dioxanes, $\left.95{ }^{\circ} \mathrm{C}\right)$ of the diboron followed by basic hydrogen peroxide oxidation $\left(3 \mathrm{M} \mathrm{NaOH}, \mathrm{H}_{2} \mathrm{O}_{2}\right.$, quench with $\mathrm{Na}_{2} \mathrm{~S}_{2} \mathrm{O}_{3}$ ). The resultant diol was protected as the acetonide ( $p \mathrm{TsOH}, 2$,2-dimethoxypropane, $60{ }^{\circ} \mathrm{C}$, $10 \mathrm{~min}$ ). Absolute stereochemistry of the 1,2-bis(boronate)esters was reported in Pelz, N.F.; Woodward, A.R.; Burks, H.E.; Sieber, J.D.; Morken, J.P. J. Am. Chem. Soc. 2004, 126, 16328.

\section{Representative Procedure for One-Pot, Sequential Diboration/Allylation/Oxidation of Allenes:}

An oven-dried $20 \mathrm{~mL}$ vial equipped with a stir-bar was charged with $10 \mathrm{mg}(0.01 \mathrm{mmol})_{\mathrm{Pd}_{2}}(\mathrm{dba})_{3}, 18$ $\mathrm{mg}(0.03 \mathrm{mmol})\left(R, R\right.$-xylyl-TADDOL)PNMe $\mathrm{PN}_{2}$, and $0.86 \mathrm{~mL}$ toluene under an inert atmosphere of argon in a dry-box. The purple solution was stirred until it appeared yellow (30 min $-1 \mathrm{~h}$ ). After this time, 130 $\mathrm{mg}(0.51 \mathrm{mmol})$ of bis(pinacolato)diboron was added to the solution. The solution was allowed to stir for $5 \mathrm{~min}$. Next, $50 \mathrm{mg}(0.43 \mathrm{mmol})$ of propa-1,2-dienyl-benzene was added to the solution. The vial was sealed with a screw-cap and allowed to stir for 12 hours at ambient temperature. After this time, $180 \mu \mathrm{L}$ of a $1.5 \mathrm{M}$ solution of isobutyraldeyhyde $(0.27 \mathrm{mmol})$ in toluene was added to the solution, and the reaction was allowed to stir for $12 \mathrm{~h}$. The vial was cooled to $0{ }^{\circ} \mathrm{C}$. Subsequently, $1 \mathrm{~mL}$ THF and $1 \mathrm{~mL} 3$ $\mathrm{M} \mathrm{NaOH}$ were added, followed by dropwise addition of $1 \mathrm{~mL}$ of a $30 \%$ aqueous $\mathrm{H}_{2} \mathrm{O}_{2}$ solution. The solution was allowed to warm to room temperature and stirred for $5 \mathrm{~h}$. The reaction was quenched with 1 $\mathrm{mL}$ of saturated aqueous $\mathrm{Na}_{2} \mathrm{~S}_{2} \mathrm{O}_{3}$ and $1 \mathrm{~mL}$ of $1 \mathrm{M} \mathrm{NaOH}$. The mixture was extracted with ethyl acetate $(2 \times 5 \mathrm{~mL})$. The organic layers were dried over anhydrous $\mathrm{Na}_{2} \mathrm{SO}_{4}$, filtered, and the solvent removed by rotary evaporation. The crude material was purified by silica gel chromatography (5:1 hexanes:ethyl acetate) to provide $51 \mathrm{mg}(93 \%)$ of 4-hydroxy-5-methyl-1-phenylhexan-2-one as a clear oil.

\section{Procedure for One-pot Simultaneous Diboration/Allylation:}

An oven-dried $20 \mathrm{~mL}$ vial equipped with a stir-bar was charged with $10 \mathrm{mg}(0.01 \mathrm{mmol}) \mathrm{Pd}_{2}(\mathrm{dba})_{3}, 18$ $\mathrm{mg}(0.03 \mathrm{mmol})\left(R, R\right.$-xylyl-TADDOL)PNMe $\mathrm{P}_{2}$, and $0.86 \mathrm{~mL}$ toluene under an inert atmosphere of argon in a dry-box. The purple solution was stirred until it appeared yellow (30 minutes -1 hour). After this time, $130 \mathrm{mg}(0.51 \mathrm{mmol})$ of bis(pinacolato)diboron was added to the solution. The solution was allowed to stir for $5 \mathrm{~min}$. Next, $50 \mathrm{mg}(0.43 \mathrm{mmol})$ of propa-1,2-dienyl-benzene and $180 \mu \mathrm{L}$ of a $1.5 \mathrm{M}$ solution of isobutyraldeyhyde $(0.27 \mathrm{mmol})$ in toluene were added to the solution, and the reaction was allowed to stir for $12 \mathrm{~h}$. The vial was cooled to $0{ }^{\circ} \mathrm{C}$. Subsequently, $1 \mathrm{~mL}$ THF and $1 \mathrm{~mL} 3 \mathrm{M} \mathrm{NaOH}$ were added, followed by dropwise addition of $1 \mathrm{~mL}$ of a $30 \%$ aqueous $\mathrm{H}_{2} \mathrm{O}_{2}$ solution. The solution was allowed to warm to room temperature and stirred for 5 hours. The reaction was quenched with $1 \mathrm{~mL}$ of saturated aqueous $\mathrm{Na}_{2} \mathrm{~S}_{2} \mathrm{O}_{3}$ and $1 \mathrm{~mL}$ of $1 \mathrm{M} \mathrm{NaOH}$. The mixture was extracted with ethyl acetate $(2 \times 5 \mathrm{~mL})$. The 
organic layers were dried over anhydrous $\mathrm{Na}_{2} \mathrm{SO}_{4}$, filtered, and the solvent removed by rotary evaporation. The crude material was purified by silica gel chromatography (5:1 hexanes:ethyl acetate) to provide $44 \mathrm{mg}(80 \%)$ of 4-hydroxy-5-methyl-1-phenylhexan-2-one as a clear oil.<smiles>CCC[C@H](O)CC(=O)Cc1ccccc1</smiles>

(R)-4-Hydroxy-1-phenylheptan-2-one. Clear oil. $\mathrm{R}_{\mathrm{f}}=0.25$ in $5: 1$ hexanes/ethyl acetate. IR (neat) 3454 (br, m), 2960 (m), $1710(\mathrm{~s}), 700.4(\mathrm{~m}) \mathrm{cm}^{-}$ ${ }^{1}$; $1 \mathrm{H}$ NMR $\left(400 \mathrm{MHz}, \mathrm{CDCl}_{3}\right): \delta 7.34-7.30(2 \mathrm{H}, \mathrm{m}), 7.28-7.24(1 \mathrm{H}, \mathrm{m}), 7.17$ $(2 \mathrm{H}, \mathrm{d}, 7.2 \mathrm{~Hz}), 4.00(1 \mathrm{H}, \mathrm{m}), 3.69(2 \mathrm{H}, \mathrm{s}), 2.92(1 \mathrm{H}, \mathrm{s}), 2.62(1 \mathrm{H}, \mathrm{dd}, J=17.6,2.8 \mathrm{~Hz}), 2.52(1 \mathrm{H}, \mathrm{dd}, J=$ $17.6,8.8 \mathrm{~Hz}), 1.44-1.36(2 \mathrm{H}, \mathrm{m}), 1.33-1.24(2 \mathrm{H}, \mathrm{m}), 0.873(3 \mathrm{H}, \mathrm{t}, J=7.0 \mathrm{~Hz}) ;{ }^{13} \mathrm{C}$ NMR $(100 \mathrm{MHz}$, $\mathrm{CDCl}_{3}$ ): $\delta 209.7,113.6,129.4,128.8,127.2,67.3,50.8,48.3,38.5,18.6,13.9$; LRMS (ESI) Calc'd for $\mathrm{C}_{13} \mathrm{H}_{18} \mathrm{O}_{2}(\mathrm{M}+\mathrm{Na})^{+}: 229.1$ Found $(\mathrm{M}+\mathrm{Na})^{+}: 229.2$.

SFC (Chiracel, AD-H, Supelco, $150 \mathrm{psi}, 50{ }^{\circ} \mathrm{C}$, flow $=3.0 \mathrm{~mL} / \mathrm{min}, 2.0 \% \mathrm{MeOH}$ ) analysis of benzoate derivative of allylboration product:

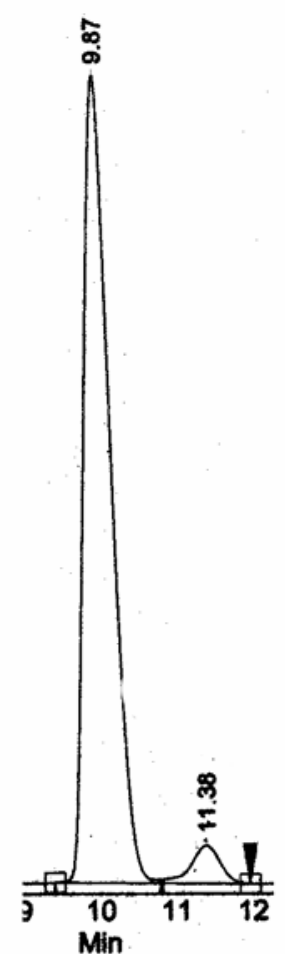

Diboration/Allylboration

Product

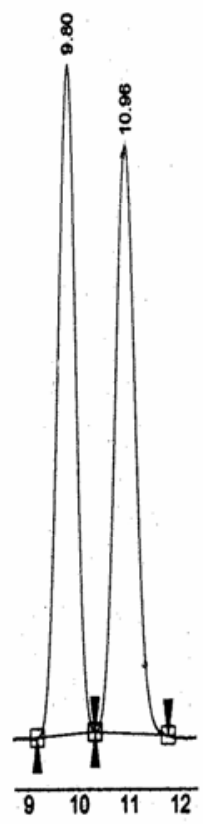

Racemic

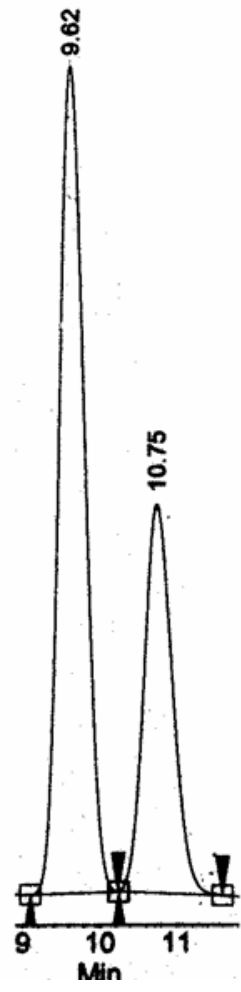

Diboration/Allylboration Product + Racemic 


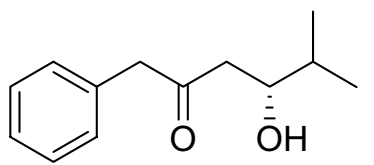

(R)-4-Hydroxy-5-methyl-1-phenylhexan-2-one. Clear oil. $\mathrm{R}_{\mathrm{f}}=0.25$ in 5:1 hexanes/ethyl acetate. IR (neat) $3450(\mathrm{~m}, \mathrm{br}), 2962(\mathrm{~m}), 1710(\mathrm{~s}), 700(\mathrm{~s}) \mathrm{cm}^{-1} ; 1 \mathrm{H}$ NMR (400 MHz, $\left.\mathrm{CDCl}_{3}\right): \delta 7.34-7.31(2 \mathrm{H}, \mathrm{m}), 7.28-7.24(1 \mathrm{H}, \mathrm{m}), 7.19-7.17$ $(2 \mathrm{H}, \mathrm{m}), 3.75(1 \mathrm{H}, \mathrm{m}), 3.71(2 \mathrm{H}, \mathrm{s}), 2.83(1 \mathrm{H}, \mathrm{s}), 2.62(1 \mathrm{H}, \mathrm{dd}, J=17.4,2.6 \mathrm{~Hz})$, $2.52(1 \mathrm{H}, \mathrm{dd}, J=17.6,9.6 \mathrm{~Hz}), 1.62(1 \mathrm{H}, \mathrm{m}), 0.86(6 \mathrm{H}, \mathrm{dd}, J=13.6,0.86 \mathrm{~Hz}) ;{ }^{13} \mathrm{C}$ NMR $(100 \mathrm{MHz}$, $\mathrm{CDCl}_{3}$ ): $\delta 209.9,133.7,129.5,128.8,127.2,72.4,50.9,45.4,33.0,18.4,17.7$; LRMS (ESI) Calc'd for $\mathrm{C}_{13} \mathrm{H}_{18} \mathrm{O}_{2}(\mathrm{M}+\mathrm{Na})^{+}: 229.1$ Found $(\mathrm{M}+\mathrm{Na})^{+}: 229.0$.

SFC (Chiracel AD-H, Supelco, $150 \mathrm{psi}, 50^{\circ} \mathrm{C}$, flow $=2.5 \mathrm{~mL} / \mathrm{min}, 2.0 \% \mathrm{MeOH}$ ) analysis of benzoate derivative of allyboration product:

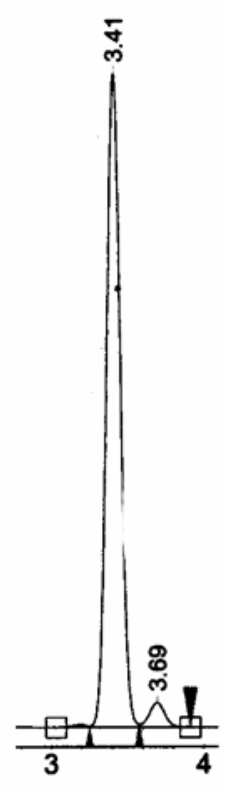

Diboration/Allylboration

Product

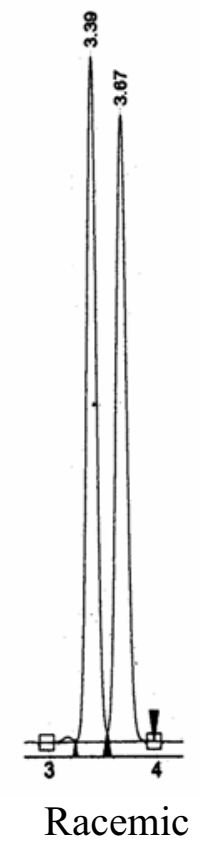

Racemic

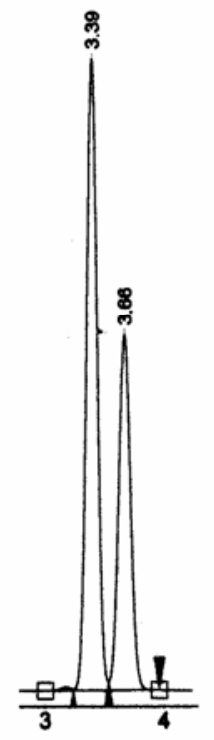

Diboration/Allylboration

Product + Racemic 
$\mathrm{C}_{10} \mathrm{H}_{21}(\boldsymbol{R})$-4-Hydroxyheptadecan-6-one. White solid. $\mathrm{R}_{\mathrm{f}}=0.38$ in 5:1 hexanes/ethyl acetate. IR $\left(\mathrm{CH}_{2} \mathrm{Cl}_{2}\right) 3056(\mathrm{~m}, \mathrm{br}), 2929(\mathrm{~s}), 2856(\mathrm{~s}), 2306(\mathrm{~m}), 1704(\mathrm{~s}) \mathrm{cm}^{-1}$;

${ }^{1} \mathrm{H}$ NMR $\left(400 \mathrm{MHz}, \mathrm{CDCl}_{3}\right): \delta 4.03-3.99(1 \mathrm{H}, \mathrm{m}), 2.56(1 \mathrm{H}, \mathrm{dd}, J=17.2,2.8 \mathrm{~Hz}), 2.46(1 \mathrm{H}, \mathrm{dd}, J=$

17.2, $9.0 \mathrm{~Hz}), 2.38(2 \mathrm{H}, \mathrm{t}, J=7.4 \mathrm{~Hz}), 1.55-1.52(2 \mathrm{H}, \mathrm{m}), 1.49-1.38(2 \mathrm{H}, \mathrm{m}), 1.36-1.28(2 \mathrm{H}, \mathrm{m}), 1.28$

- 1. $24(16 \mathrm{H}, \mathrm{m}), 1.32(3 \mathrm{H}, \mathrm{t}, \mathrm{J}=6.6 \mathrm{~Hz}), 1.28(3 \mathrm{H}, \mathrm{t}, J=6.4 \mathrm{~Hz}) ;{ }^{13} \mathrm{C} \mathrm{NMR}\left(100 \mathrm{MHz}, \mathrm{CDCl}_{3}\right): \delta 213.0$,

67.7, 49.3, 44.1, 39.0, 32.3, 30.0, 29.8, 29.7, 29.6, 24.0, 23.1, 19.1, 14.5, 14.4; LRMS (ESI) Calc'd for

$\mathrm{C}_{13} \mathrm{H}_{24} \mathrm{O}_{2}(\mathrm{M}+\mathrm{Na})^{+}: 293.3$ Found $(\mathrm{M}+\mathrm{Na})^{+}: 293.3$.

SFC (Chiracel AD-H, Supelco, $150 \mathrm{psi}, 50^{\circ} \mathrm{C}$, flow $=2.0 \mathrm{~mL} / \mathrm{min}, 2.0 \% \mathrm{MeOH}$ ) analysis of benzoate derivative of allylboration product:

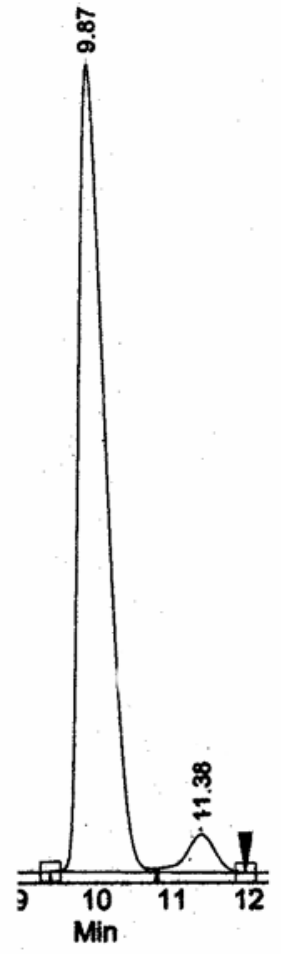

Diboration/Allylboration

Product

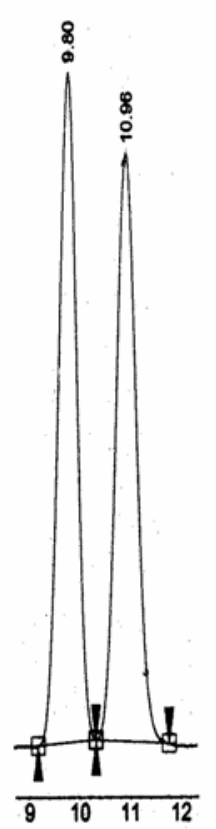

Racemic

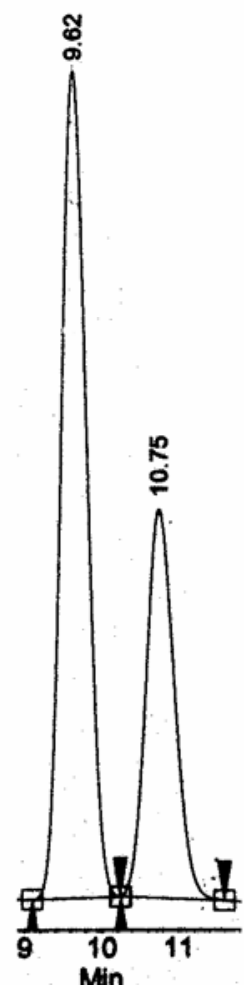

Diboration/Allylboration

Product + Racemic 


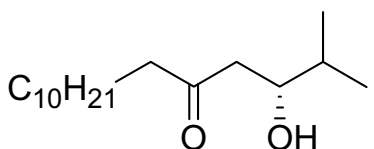

(R)-3-Hydroxy-2-methylhexadecan-5-one. White solid. $\mathrm{R}_{\mathrm{f}}=0.47$ in 5:1

hexanes/ethyl acetate. IR $\left(\mathrm{CH}_{2} \mathrm{Cl}_{2}\right) 2925(\mathrm{~s}), 2858(\mathrm{~s}), 1704(\mathrm{~s}), 1470(\mathrm{~m}), 1370$

(m) $\mathrm{cm}^{-1} ;{ }^{1} \mathrm{H}$ NMR $\left(400 \mathrm{MHz}, \mathrm{CDCl}_{3}\right): \delta 3.80(1 \mathrm{H}, \mathrm{ddd}, J=9.2,6.0,2.4 \mathrm{~Hz}), 2.58$ $1.59-1.55(2 \mathrm{H}, \mathrm{m}), 1.25(18 \mathrm{H}, \mathrm{s}, \mathrm{br}), 0.92(6 \mathrm{H}, \mathrm{dd}, \mathrm{J}=10.8,6.8 \mathrm{~Hz}), 0.88(3 \mathrm{H}, \mathrm{t}, \mathrm{J}=7.0 \mathrm{~Hz}) ;{ }^{13} \mathrm{C} \mathrm{NMR}$ $\left(100 \mathrm{MHz}, \mathrm{CDCl}_{3}\right): \delta 213.3,72.7,46.3,44.2,33.4,32.3,30.0,29.9,29.8,29.7,29.6,24.0,23.1,18.8$, 18.2, 14.5; LRMS (ESI) Calc'd for $\mathrm{C}_{13} \mathrm{H}_{24} \mathrm{O}_{2}(\mathrm{M}+\mathrm{H})^{+}: 271.3$ Found $(\mathrm{M}+\mathrm{H})^{+}: 271.3$.

Proof of Stereochemistry. Absolute stereochemistry was established in comparison to material prepared by reaction of isobutyraldehyde with the boron enolate-derived from chlorodiisocampheylborane and tridecan-2-one. The reaction provided the methylketone aldol adduct (S)-3-Hydroxy-2-methylhexadecan5-one in 65\% ee according to literature procedures [(a) Paterson, I.; Goodman, J. M.; Lister, M. A.; Schumann, R. C.; McClure, C. K.; Norcross, R. D. Tetrahedron 1990, 46, 4663. (b) Brown, H. C., Dhar, R. K., Ganesan, K., Singaram, B. J. Org.Chem. 1992, 57, 499.].

SFC (Chiracel AD-H, $150 \mathrm{psi}, 50{ }^{\circ} \mathrm{C}$, flow $=3.0 \mathrm{~mL} / \mathrm{min}, 4.0 \% \mathrm{MeOH}$ ) analysis of benzoate derivative of allylboration product (retention times shown in minutes):

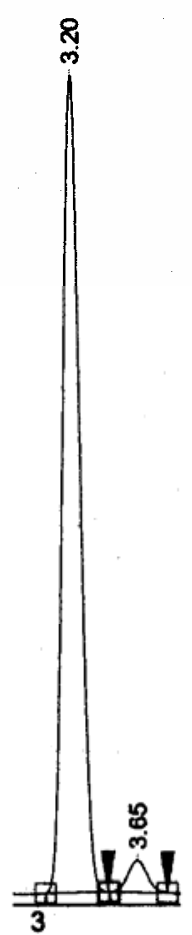

Allylboration Product

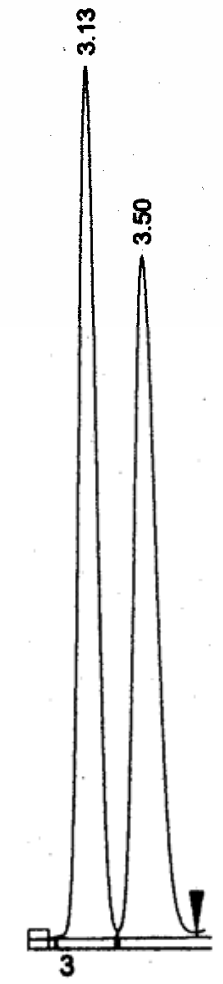

Racemic

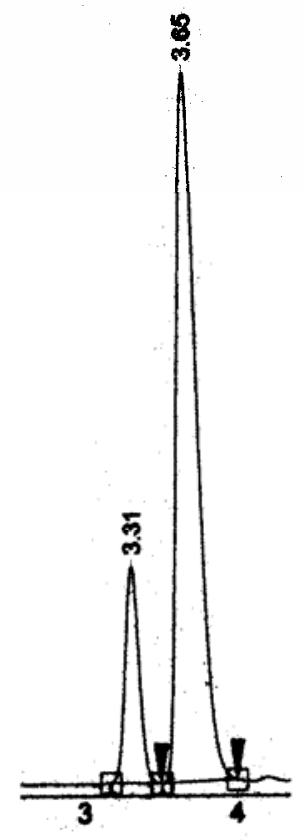

Authentic

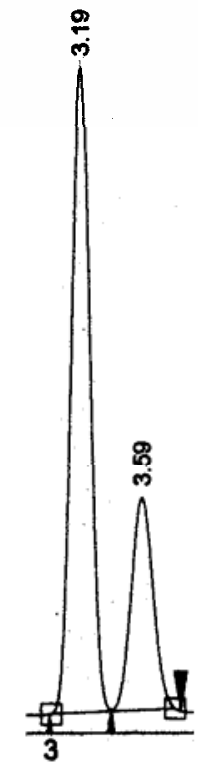

Allylboration

Product

+ Racemic 
<smiles>CCCCCCCCCC(=O)C[C@H](O)c1ccccc1</smiles>

(R)1-Hydroxy-1-phenyltetradecan-3-one. White solid. $\mathrm{R}_{\mathrm{f}}=0.34$ in 5:1 hexanes/ethyl acetate. IR $\left(\mathrm{CH}_{2} \mathrm{Cl}_{2}\right) 2927$ (s), 2858 (s), $1704(\mathrm{~m}) \mathrm{cm}^{-1} ;{ }^{1} \mathrm{H}$ NMR $\left(400 \mathrm{MHz}, \mathrm{CDCl}_{3}\right): \delta 7.33-7.30(4 \mathrm{H}, \mathrm{m}), 7.29-7.23(1 \mathrm{H}, \mathrm{m}), 5.13(1 \mathrm{H}, \mathrm{dd}$, $J=8.6,3.6 \mathrm{~Hz}), 2.86-2.80(1 \mathrm{H}, \mathrm{dd}, J=17.3,8.6 \mathrm{~Hz}), 2.79-2.74(1 \mathrm{H}, \mathrm{dd}, J$ $=17.4,3.6 \mathrm{~Hz}), 2.40(2 \mathrm{H}, \mathrm{t}, J=7.4 \mathrm{~Hz}), 1.60-1.50(2 \mathrm{H}$, br m), $1.30-1.15(16 \mathrm{H}$, br m), $0.87(3 \mathrm{H}, \mathrm{t}, J$ $=6.8 \mathrm{~Hz}) ;{ }^{13} \mathrm{C} \mathrm{NMR}\left(100 \mathrm{MHz}, \mathrm{CDCl}_{3}\right): \delta 212.1,143.3,128.9,128.0,126.0,70.4,51.4,44.2,32.3,30.0$, 29.8, 29.7, 29.5, 24.0, 23.1, 14.5; LRMS (ESI) Calc'd for $\mathrm{C}_{20} \mathrm{H}_{32} \mathrm{O}_{2}(\mathrm{M}+\mathrm{Na})^{+}: 327.5$ Found $(\mathrm{M}+\mathrm{Na})^{+}$: 327.3.

SFC (Chiracel AD-H, Supelco, $150 \mathrm{psi}, 50^{\circ} \mathrm{C}$, flow $=3.0 \mathrm{~mL} / \mathrm{min}, 4.0 \% \mathrm{MeOH}$ ) analysis of benzoate derivative of allylboration product:

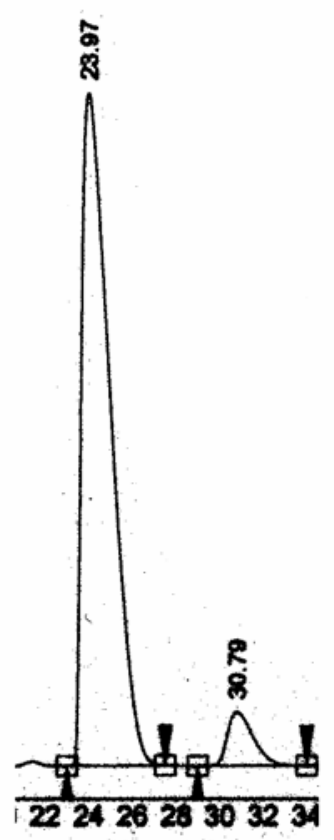

Diboration/Allylboration

Product

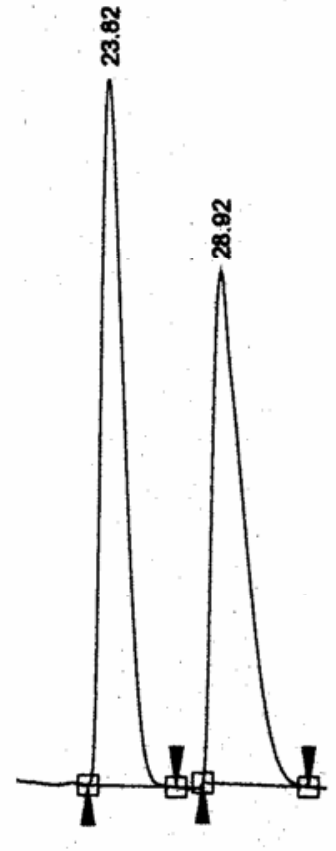

$\longdiv { 0 2 2 2 4 2 6 2 8 3 0 3 2 3 }$

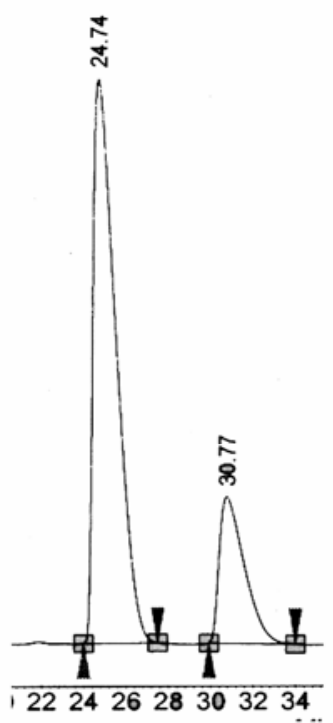

Diboration/Allylboration

Product + Racemic 
$\checkmark \prod_{O}{ }_{\mathrm{O} H}$

(R)- 1-Cyclohexyl-4-hydroxyheptan-2-one. White solid. $\mathrm{R}_{\mathrm{f}}=0.23$ in 10:1 hexanes/ethyl acetate. IR (neat) 3317 (m, br), 2919 (s), 1700 (s) $\mathrm{cm}^{-1} ;{ }^{1} \mathrm{H}$ NMR $\left(400 \mathrm{MHz}, \mathrm{CDCl}_{3}\right): \delta 4.01(1 \mathrm{H}, \mathrm{m}), 3.04(1 \mathrm{H}, \mathrm{d}, J=3.2), 2.56(1 \mathrm{H}, \mathrm{dd}, J=17.6$,

$2.8 \mathrm{~Hz}), 2.45(1 \mathrm{H}, \mathrm{dd}, J=17.6,8.8 \mathrm{~Hz}), 2.27(2 \mathrm{H}, \mathrm{d}, J=6.8 \mathrm{~Hz}), 1.86-1.75(1 \mathrm{H}, \mathrm{m}), 1.69-1.59(5 \mathrm{H}$, $\mathrm{m}), 1.52-1.18(6 \mathrm{H}, \mathrm{m}), 1.17-1.06(1 \mathrm{H}, \mathrm{m}), 0.96-0.85(5 \mathrm{H}, \mathrm{m}) ;{ }^{13} \mathrm{C} \mathrm{NMR}\left(100 \mathrm{MHz}, \mathrm{CDCl}_{3}\right): \delta 212.5$, 67.3, 51.4, 49.6, 38.5, 33.9, 33.2, 26.1, 26.0, 18.6, 14.0; LRMS (ESI) Calc'd for $\mathrm{C}_{13} \mathrm{H}_{24} \mathrm{O}_{2}(\mathrm{M}+\mathrm{Na})^{+}$:

235.2 Found $(\mathrm{M}+\mathrm{Na})^{+}: 235.1$.

Proof of Stereochemistry. Absolute stereochemistry was established in comparison to material prepared by reaction of butyraldehyde with the boron enolate-derived from chlorodiisocampheylborane and 1cyclohexylpropan-2-one. The reaction provided the methylketone aldol adduct $(S)$-1-cyclohexyl-4hydroxyheptan-2-one in 48\% ee according to literature procedures [(a) Paterson, I.; Goodman, J. M.; Lister, M. A.; Schumann, R. C.; McClure, C. K.; Norcross, R. D. Tetrahedron 1990, 46, 4663. (b) Brown, H. C., Dhar, R. K., Ganesan, K., Singaram, B. J. Org.Chem. 1992, 57, 499.].

SFC (Chiracel AD-H, Supelco, $150 \mathrm{psi}, 50^{\circ} \mathrm{C}$, flow $=2.5 \mathrm{~mL} / \mathrm{min}, 2.0 \% \mathrm{MeOH}$ ) analysis of benzoate derivative of allylboration product:

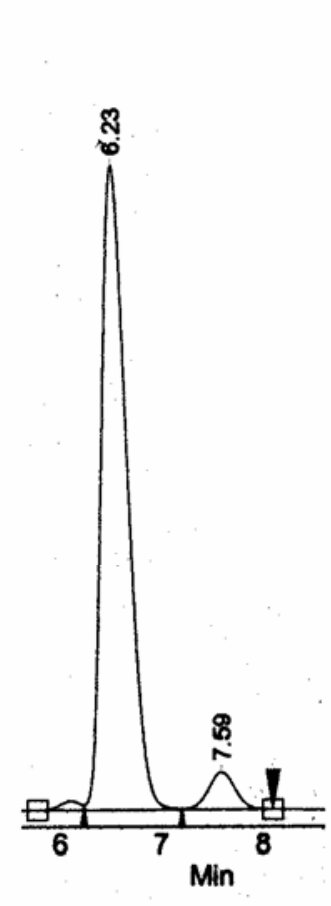

Diboration Product

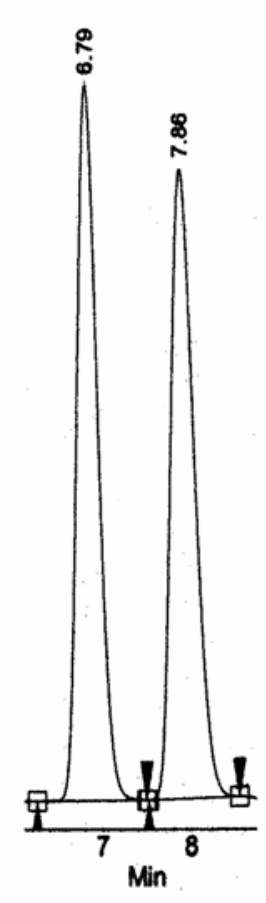

Racemic

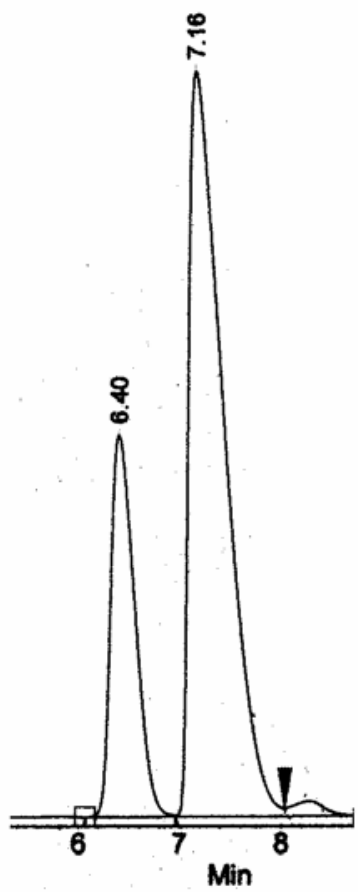

Authentic

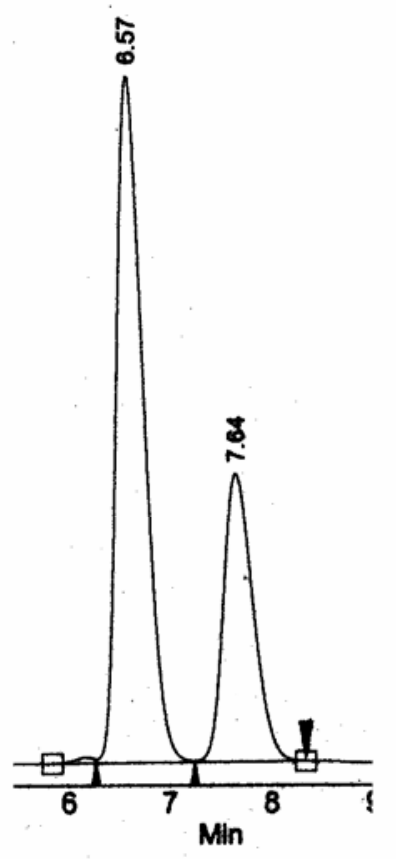

Diboration Product + Racemic 


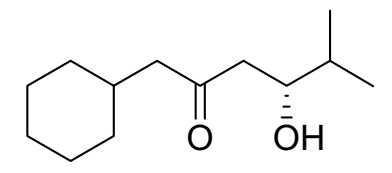

(R)-1-Cyclohexyl-4-hydroxy-5-methylhexan-2-one. Clear oil. $R_{\mathrm{f}}=0.29$ in 10:1 hexanes/ethyl acetate. IR (neat) 3491 (br, m) 2925 (s), 1702 (m) $\mathrm{cm}^{-1}$; ${ }^{1} \mathrm{H}$ NMR $\left(400 \mathrm{MHz}, \mathrm{CDCl}_{3}\right): \delta 3.77(1 \mathrm{H}, \mathrm{m}) 3.0(1 \mathrm{H}, \mathrm{d}, J=2.4 \mathrm{~Hz}) 2.53(1 \mathrm{H}, \mathrm{dd}, J=17.2$, $2.6 \mathrm{~Hz}), 2.43(1 \mathrm{H}, \mathrm{dd}, J=17.2,9.6 \mathrm{~Hz}), 2.28(2 \mathrm{H}, \mathrm{d}, J=6.8 \mathrm{~Hz}), 1.83-1.76(1 \mathrm{H}$, m), $1.66-1.59(6 \mathrm{H}, \mathrm{m}), 1.24-1.91(2 \mathrm{H}, \mathrm{m}), 1.16-1.09(2 \mathrm{H}, \mathrm{m}), 0.94-0.87(8 \mathrm{H}, \mathrm{m}) ;{ }^{13} \mathrm{C}$ NMR $(100$ $\left.\mathrm{MHz}, \mathrm{CDCl}_{3}\right): \delta 212.6,72.2,51.4,46.6,33.9,33.2,33.0,26.2,26.0,18.4,17.8$; LRMS (ESI) Calc'd for $\mathrm{C}_{13} \mathrm{H}_{24} \mathrm{O}_{2}(\mathrm{M}+\mathrm{H})^{+}: 213.2$ Found $(\mathrm{M}+\mathrm{H})^{+}: 213.2$.

SFC (Chiracel OD-H, Supelco, $150 \mathrm{psi}, 50{ }^{\circ} \mathrm{C}$, flow $=2.0 \mathrm{~mL} / \mathrm{min}, 1.0 \% \mathrm{MeOH}$ ) analysis of benzoate derivative of allylboration product:

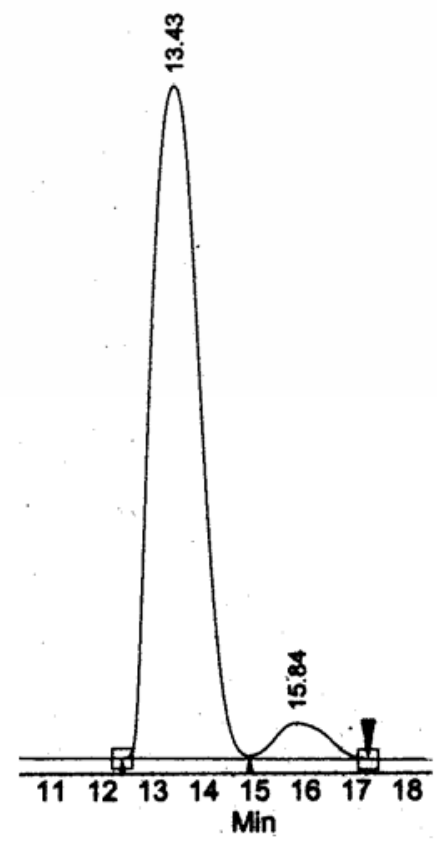

Allylboration Product

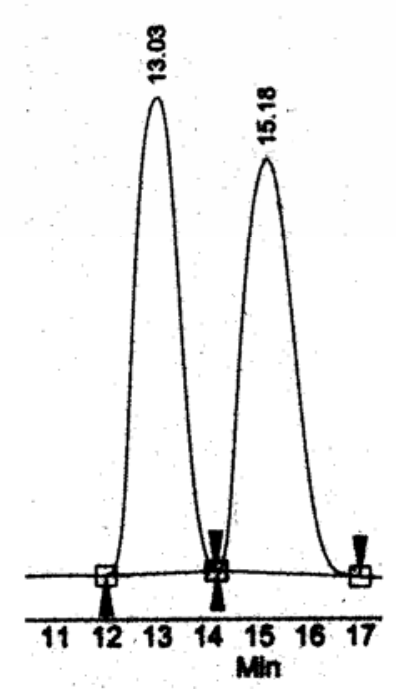

Racemic

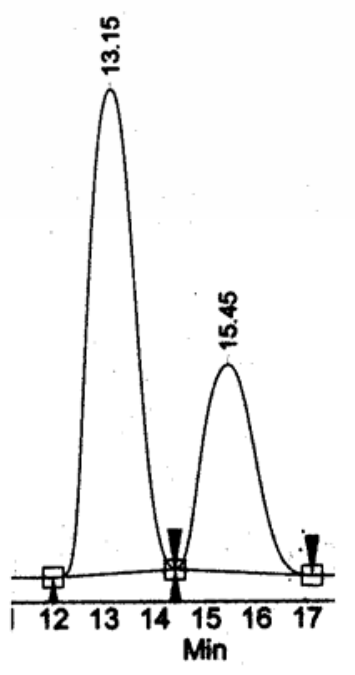

Allylboration Product +

Racemic 
<smiles>O=C(CC1CCCCC1)C[C@@H](O)c1ccccc1</smiles>
$1.77(1 \mathrm{H}, \mathrm{m}), 1.68-1.62(5 \mathrm{H}, \mathrm{m}), 1.30-1.08(3 \mathrm{H}, \mathrm{m}), 0.95-0.85(2 \mathrm{H}, \mathrm{m}) ;{ }^{13} \mathrm{C} \mathrm{NMR}(100 \mathrm{MHz}$, $\left.\mathrm{CDCl}_{3}\right): \delta 211.4,142.8,128.4,127.5,125.6,69.8,51.6,51.4,33.8,33.1,26.1,26.0$; LRMS (ESI) Calc'd for $\mathrm{C}_{16} \mathrm{H}_{22} \mathrm{O}_{2}(\mathrm{M}+\mathrm{Na})^{+}:$: 269.3 Found $(\mathrm{M}+\mathrm{Na})^{+}: 269.1$.

SFC (Chiracel AD-H, Supelco, $150 \mathrm{psi}, 50{ }^{\circ} \mathrm{C}$, flow $=3.0 \mathrm{~mL} / \mathrm{min}, 2.0 \% \mathrm{MeOH}$ ) analysis of benzoate derivate of allylboration product:

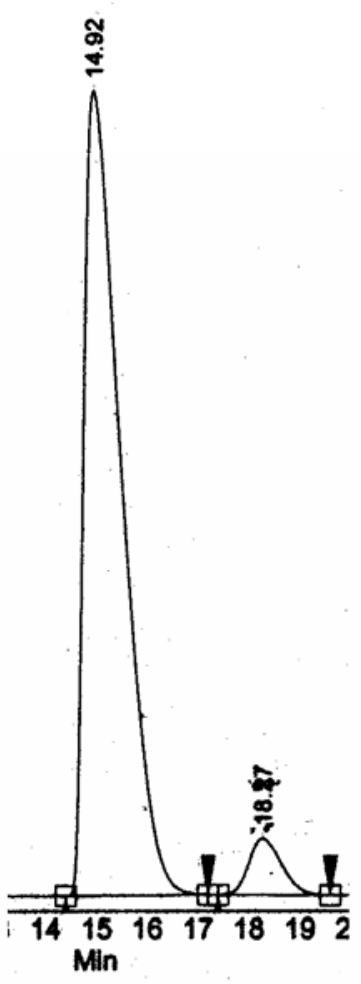

Diboration/Allylboration

Product

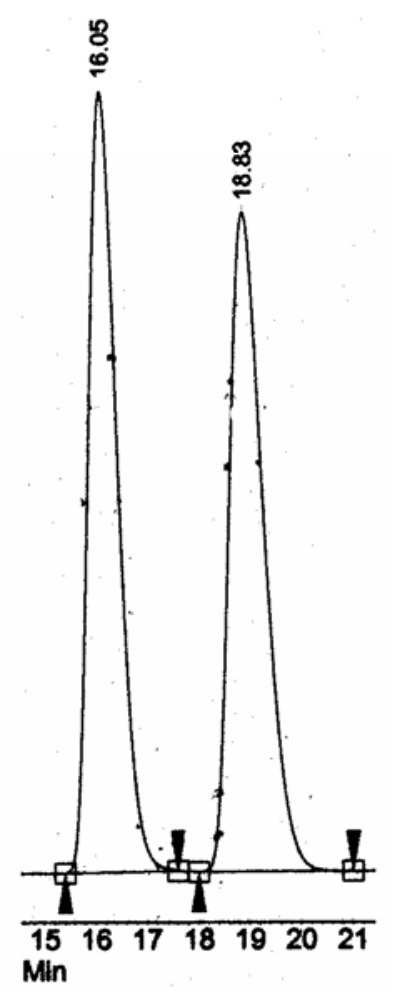

Racemic

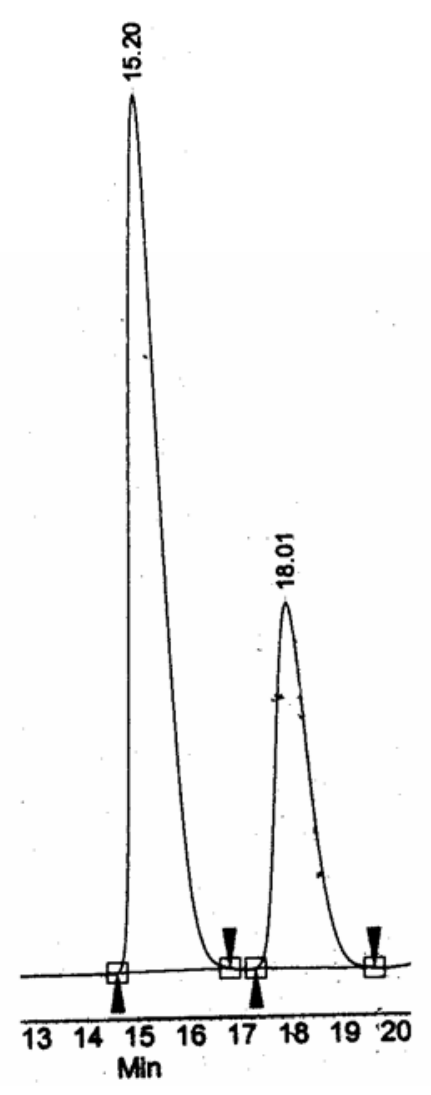

Diboration/Allylboration

Product + Racemic 


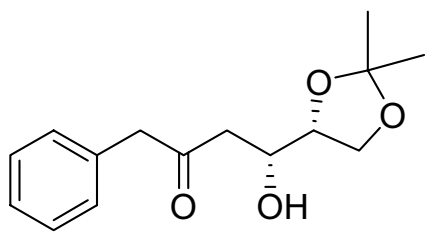
s), $2.71(1 \mathrm{H}, \mathrm{dd}, J=17.0,8.2 \mathrm{~Hz}), 2.59(1 \mathrm{H}, \mathrm{dd}, J=17.0,3.4 \mathrm{~Hz}), 1.37(6 \mathrm{H}, \mathrm{d}, J=30.4 \mathrm{~Hz}) ;{ }^{13} \mathrm{C}$ NMR $\left(100 \mathrm{MHz}, \mathrm{CDCl}_{3}\right): \delta 208.2,134.0,129.9,129.2,127.6,110.0,78.1,66.3,65.9,51.3,45.2,26.7,25.5$; LRMS (ESI) Calc'd for $\mathrm{C}_{15} \mathrm{H}_{20} \mathrm{O}_{4}(\mathrm{M}+\mathrm{Na})^{+}: 287.1$ Found $(\mathrm{M}+\mathrm{Na})^{+}$: 287.2.

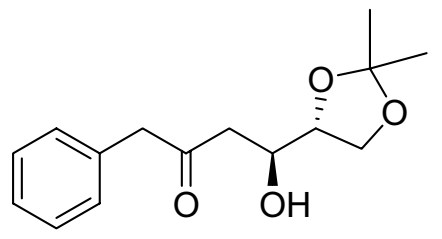

\section{4-(2,2-Dimethyl-[1,3]dioxolan-4S-yl)-4S-hydroxy-1-phenylbutan-2-one} (5). Clear oil. $\mathrm{R}_{\mathrm{f}}=0.51$ in 1:1 hexanes/ethyl acetate. IR $\left(\mathrm{CH}_{2} \mathrm{Cl}_{2}\right): 3570(\mathrm{~s})$, 2896 (s), 1725 (s), 1497 (m) $1382(\mathrm{~m}), 1206(\mathrm{~m}), 1075(\mathrm{~s}) \mathrm{cm}^{-1} ;{ }^{1} \mathrm{H}$ NMR $\left(400 \mathrm{MHz}, \mathrm{CDCl}_{3}\right): \delta 7.36-7.32(2 \mathrm{H}, \mathrm{m}), 7.30-7.26(1 \mathrm{H}, \mathrm{m}), 7.21-7.19$ $(2 \mathrm{H}, \mathrm{m}), 4.06-4.01(1 \mathrm{H}, \mathrm{m}), 3.97-3.88(3 \mathrm{H}, \mathrm{m}), 3.74(2 \mathrm{H}, \mathrm{s}), 2.85(1 \mathrm{H}, \mathrm{dd}$, $J=17.8,2.6 \mathrm{~Hz}), 2.63(1 \mathrm{H}, \mathrm{dd}, J=18.0,8.4 \mathrm{~Hz}), 1.34(6 \mathrm{H}, \mathrm{d}, J=15.6 \mathrm{~Hz}) ;{ }^{13} \mathrm{C}$ NMR $(100 \mathrm{MHz}$, $\mathrm{CDCl}_{3}$ ): $\delta$ 209.7, 133.8, 129.9, 129.2, 127.7, 109.9, 69.5, 67.2, 51.2, 45.2, 27.0, 25.6; LRMS (ESI) Calc'd for $\mathrm{C}_{15} \mathrm{H}_{20} \mathrm{O}_{4}(\mathrm{M}+\mathrm{Na})^{+}:$287.1 Found $(\mathrm{M}+\mathrm{Na})^{+}: 287.2$.

The diastereomer ratio of the reaction products in Scheme 2 was determined by ${ }^{1} \mathrm{H}$ NMR analysis of the unpurified reaction mixture. The relative configuration was established in analogy to the reaction products described herein and by comparison to similar glyceraldehyde-derived methyl ketone aldol adducts. For comparison, relevant data is below. Note that for the syn diastereomer the downfield indicated methylene proton always has a larger vicinal coupling with the adjacent carbinol (ca. $8 \mathrm{~Hz})$ versus the upfield methylene proton $(\mathrm{ca} .3 \mathrm{~Hz}$ ). For the anti diastereomer, this situation is reversed.

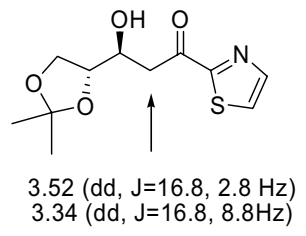

Dondoni, A.; Merino, P. J. Org. Chem. 1991, 56, 5294

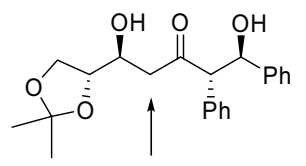

$2.75(\mathrm{dd}, \mathrm{J}=16.8,2.9 \mathrm{~Hz})$

$2.64(\mathrm{dd}, \mathrm{J}=16.8,8.3 \mathrm{~Hz})$

Santos et. al Tetrahedron: Asymm. 2001, 12, 3447.

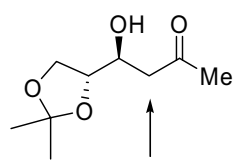

$2.75(\mathrm{dd}, \mathrm{J}=17.5,1.5 \mathrm{~Hz})$

$2.51(\mathrm{dd}, \mathrm{J}=17.5,8.2 \mathrm{~Hz})$

Garcia et. al Tetrahedron 1998, 54, 6867 similar data: Cubero Carbohydrate Research, 1986,

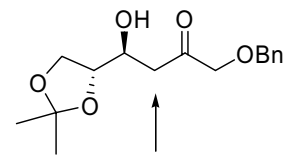

$2.85(\mathrm{dd}, \mathrm{J}=17.5,2.5 \mathrm{~Hz})$

$2.66(\mathrm{dd}, \mathrm{J}=17.5,8.5 \mathrm{~Hz})$

Gefflaut J. Org. Chem. 2001, 66, 2296.

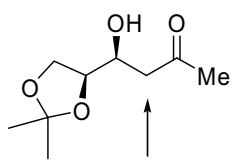

$2.72(\mathrm{dd}, \mathrm{J}=17,8 \mathrm{~Hz})$ 2.51 (dd, J=17, $3.75 \mathrm{~Hz}$ )

Cubero Carbohydrate Research, 1986, 148, 209.

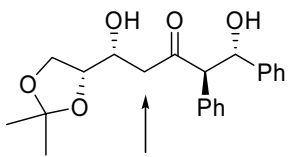

$2.74(\mathrm{dd}, \mathrm{J}=16.6,8.6 \mathrm{~Hz})$ $2.64(\mathrm{dd}, \mathrm{J}=16.6,3.0 \mathrm{~Hz})$

Santos et. al Tetrahedron: Asymm. 2001, 12, 3447. 


\section{Procedure for Diboration/Allylation/Protiodeboration:}

An oven-dried $20 \mathrm{~mL}$ vial equipped with a stir-bar was charged with $9 \mathrm{mg}(0.01 \mathrm{mmol}) \mathrm{Pd}_{2}\left(\mathrm{dba}_{3}, 16 \mathrm{mg}\right.$ $(0.25 \mathrm{mmol})\left(R, R\right.$-xylyl-TADDOL)PNMe $\mathrm{e}_{2}$, and $0.80 \mathrm{~mL}$ toluene under an inert atmosphere of argon in a dry-box. The purple solution was stirred until it appeared yellow (30 minutes -1 hour). After this time, $121 \mathrm{mg}(0.475 \mathrm{mmol})$ of bis(pinacolato)diboron was added to the solution. The solution was allowed to stir for 5 minutes. Next, $72 \mathrm{mg}(0.40 \mathrm{mmol})$ of trideca-1,2-diene and $167 \mu \mathrm{L}$ of a $1.5 \mathrm{M}$ solution of isobutyraldeyhyde $(0.250 \mathrm{mmol})$ were added to the solution, and the reaction was allowed to stir for $12 \mathrm{~h}$. The solvent was removed under reduced pressure and $0.5 \mathrm{~mL} \mathrm{CH}_{3} \mathrm{CO}_{2} \mathrm{H}$ was added. The reaction was warmed to $100{ }^{\circ} \mathrm{C}$ for 5 hours then allowed to cool to room temperature. The reaction was quenched with $2 \mathrm{~mL} 1 \mathrm{M} \mathrm{NaOH}$ and extracted with ethyl acetate $(2 \times 5 \mathrm{~mL})$. The organic layers were dried over anhydrous $\mathrm{Na}_{2} \mathrm{SO}_{4}$, filtered, and the was solvent removed by rotary evaporation. The crude material was purified by silica gel chromatography (10:1 hexanes/ethyl acetate) to provide $52 \mathrm{mg}(82 \%)$ of 2methylhexadec-5-en-3-ol as a clear oil.

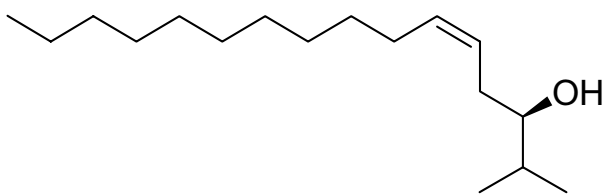

2-Methylhexadec-5Z-en-3-ol (7). Clear oil. $\mathrm{R}_{\mathrm{f}}=0.45$ in 10:1 hexanes/ethyl acetate. IR $\left(\mathrm{CH}_{2} \mathrm{Cl}_{2}\right): 2929$ (s), $2856(\mathrm{~s}), 1735$ (m), $1468(\mathrm{~m}) \mathrm{cm}^{-1} ;{ }^{1} \mathrm{H}$ NMR $\left(400 \mathrm{MHz}, \mathrm{CDCl}_{3}\right): \delta 5.60-5.54(1 \mathrm{H}, \mathrm{m})$, $5.44-5.38(1 \mathrm{H}, \mathrm{m}), 3.39-3.34(1 \mathrm{H}, \mathrm{m}), 2.25-2.17(2 \mathrm{H}, \mathrm{m}), 2.08$ - $1.99(2 \mathrm{H}, \mathrm{m}), 1.74-1.63(1 \mathrm{H}, \mathrm{m}), 1.25(16 \mathrm{H}, \mathrm{br} \mathrm{s}), 0.94(6 \mathrm{H}, \mathrm{dd}, J=6.8,4.8 \mathrm{~Hz}), 0.89(3 \mathrm{H}, \mathrm{t}, J=6.8$ $\mathrm{Hz}) ;{ }^{13} \mathrm{C}$ NMR $\left(100 \mathrm{MHz}, \mathrm{CDCl}_{3}\right): \delta 134.0,125.9,76.6,33.5,32.6,32.3,30.1,30.0,29.9,29.7,27.8$, 23.1, 19.2, 18.0, 14.5; LRMS (ESI) Calc'd for $\mathrm{C}_{17} \mathrm{H}_{34} \mathrm{O}(\mathrm{M}+\mathrm{Na})^{+}: 277.5$ Found $(\mathrm{M}+\mathrm{Na})^{+}: 277.3$.

Olefin geometry was determined by analysis of the vinylic ${ }^{1} \mathrm{H}-{ }^{1} \mathrm{H}$ coupling constant after decoupling of the allylic protons $(\mathrm{J}=10.8 \mathrm{~Hz})$. Enantiomeric excess was determined by ${ }^{1} \mathrm{H}$ NMR analysis of the Mosher ester.
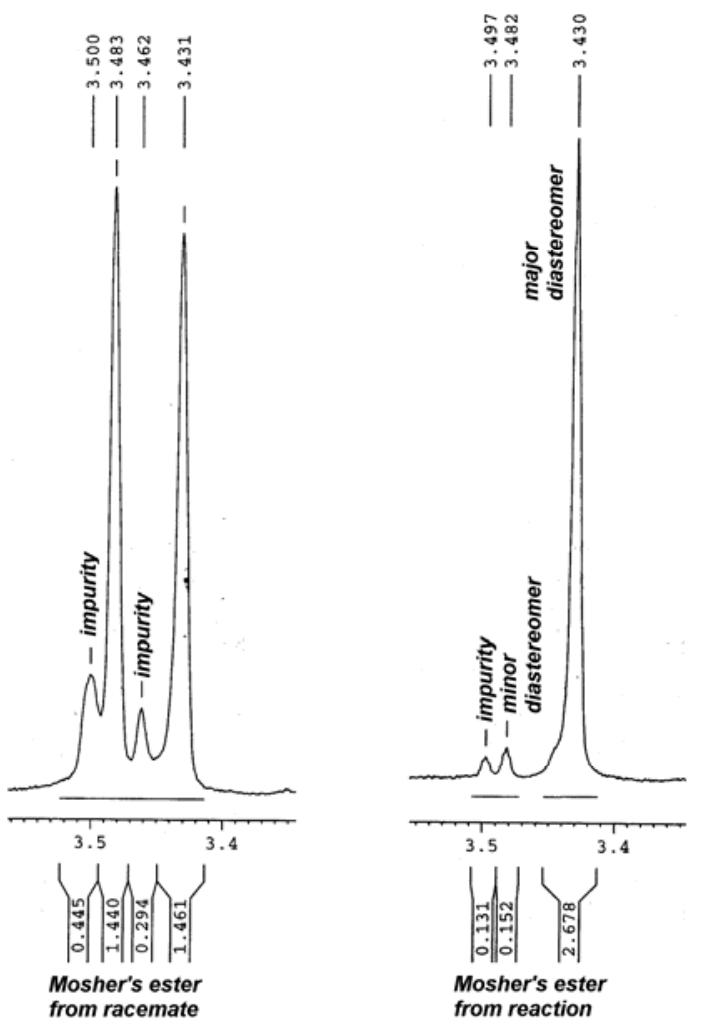

Page S- 13 


\section{Procedure for Diboration/Allylation/Iodination:}

An oven-dried $20 \mathrm{~mL}$ vial equipped with a stir-bar was charged with $9 \mathrm{mg}(0.01 \mathrm{mmol}) \mathrm{Pd}_{2}(\mathrm{dba})_{3}, 16 \mathrm{mg}$ $(0.25 \mathrm{mmol})(R, R$-xylyl-TADDOL)PNMe 2 , and $0.80 \mathrm{~mL}$ toluene under an inert atmosphere of argon in a dry-box. The purple solution was stirred until it appeared yellow (30 minutes -1 hour). After this time, $121 \mathrm{mg}(0.475 \mathrm{mmol})$ of bis(pinacolato)diboron was added to the solution. The solution was allowed to stir for 5 minutes. Next, $72 \mathrm{mg}(0.40 \mathrm{mmol})$ of trideca-1,2-diene and $167 \mu \mathrm{L}$ of a $1.5 \mathrm{M}$ solution of isobutyraldeyhyde $(0.250 \mathrm{mmol})$ were added to the solution, and the reaction was allowed to stir for $12 \mathrm{~h}$. The solvent was removed under reduced pressure and the crude material was filtered through a plug of silica with $10: 1$ hexanes/ethyl acetate. THF $(0.5 \mathrm{~mL})$ and $3 \mathrm{M} \mathrm{NaOH}(0.25 \mathrm{~mL})$ were then added. A solution of $0.5 \mathrm{M} \mathrm{I}_{2}(1.0 \mathrm{~mL})$ was added dropwise over 30 minutes. The reaction was diluted with $2 \mathrm{~mL}$ $\mathrm{H}_{2} \mathrm{O}$ and extracted with ethyl acetate $(2 \times 5 \mathrm{~mL})$. The organics were dried over $\mathrm{Na}_{2} \mathrm{SO}_{4}$, filtered, and the solvent was removed by rotary evaporation. The crude material was purified by silica gel chromatography (15:1 hexanes/ethyl acetate) to provide $45 \mathrm{mg}$ (47\%) of 5E-iodo-2-methylhexadec-5-en$3 R$-ol as a clear oil.

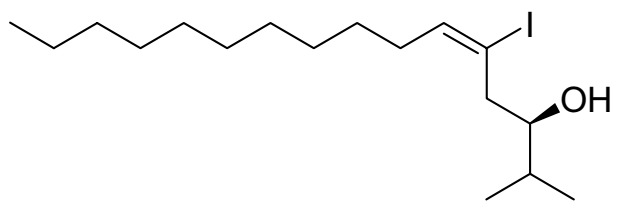

5E-Iodo-2-methylhexadec-5-en-3R-ol (6). Clear oil. $\mathrm{R}_{\mathrm{f}}=0.47$ in 10:1 hexanes/ethyl acetate. IR $\left(\mathrm{CH}_{2} \mathrm{Cl}_{2}\right) 3595(\mathrm{~m}), 2927$ (s), 2858 (s), $563(\mathrm{~s}) \mathrm{cm}^{-1} ;{ }^{1} \mathrm{H}$ NMR $\left(400 \mathrm{MHz}, \mathrm{CDCl}_{3}\right): \delta 6.38(1 \mathrm{H}, \mathrm{t}, J=7.6$ $\mathrm{Hz}), 3.68-3.63(1 \mathrm{H}, \mathrm{m}), 2.64(1 \mathrm{H}, \mathrm{dd}, J=14.4,9.2 \mathrm{~Hz}), 2.39(1 \mathrm{H}$, dd, $J=14.4,2.2 \mathrm{~Hz}), 2.13-2.06(2 \mathrm{H}, \mathrm{m}), 1.79-1.72(1 \mathrm{H}, \mathrm{m}), 1.37-1.33(2 \mathrm{H}, \mathrm{m}), 1.26(15 \mathrm{H}, \mathrm{br}), 0.97$ $(6 \mathrm{H}, \mathrm{dd}, J=6.8,5.2 \mathrm{~Hz}), 0.88(3 \mathrm{H}, \mathrm{t}, J=7.0) ;{ }^{13} \mathrm{C}$ NMR $\left(125 \mathrm{MHz}, \mathrm{CDCl}_{3}\right): \delta 144.9,98.7,74.8,43.1$, $32.6,31.8,31.2,29.5,29.4,29.3,29.2,29.0,22.6,18.7,17.6$, 14.0; LRMS (ESI) Calc'd for $\mathrm{C}_{17} \mathrm{H}_{33} \mathrm{IO}$ $(\mathrm{M}+\mathrm{Na})^{+}: 403.3$ Found $(\mathrm{M}+\mathrm{Na})^{+}: 403.3$.

Alkene geometry was confirmed by treatment of the vinyl iodide with 3.0 equiv of $n$-butyllithium in hexanes and tetrahydrofuran at $-78{ }^{\circ} \mathrm{C}$ followed by $\mathrm{H}_{2} \mathrm{O}$ to give 7 . Analysis of the coupling constant between the vinylic hydrogens $(J=10.8 \mathrm{~Hz})$ indicates the cis-alkene is present. Mosher's ester analysis was performed on the dehalogenated material and compared to that for 7 above.

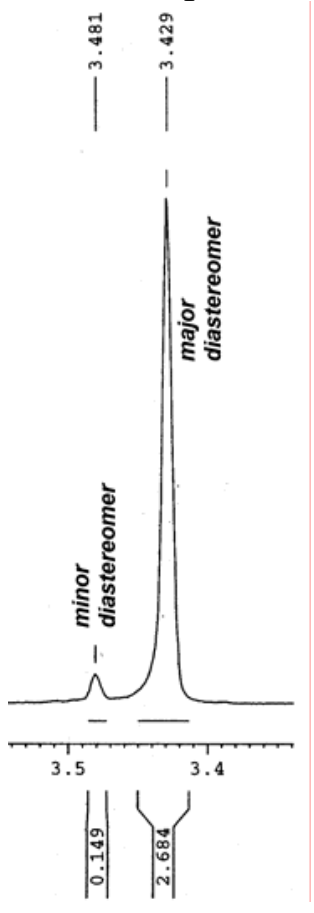

Page S- 14 


\section{Procedure for One-pot Diboration/Allylation/Suzuki Coupling:}

An oven-dried $20 \mathrm{~mL}$ vial equipped with a stir-bar was charged with $10 \mathrm{mg}(0.01 \mathrm{mmol}) \mathrm{Pd}_{2}(\mathrm{dba})_{3}, 18$ $\mathrm{mg}(0.03 \mathrm{mmol})\left(R, R\right.$-xylyl-TADDOL)PNMe $\mathrm{P}_{2}$, and $0.86 \mathrm{~mL}$ toluene under an inert atmosphere of argon in a dry-box. The purple solution was stirred until it appeared yellow (30 minutes -1 hour). After this time, $130 \mathrm{mg}(0.51 \mathrm{mmol})$ of bis(pinacolato)diboron was added to the solution. The solution was allowed to stir for 5 minutes. Next, $50 \mathrm{mg}(0.43 \mathrm{mmol})$ of propa-1,2-dienyl-benzene and $180 \mu \mathrm{L}$ of a $1.5 \mathrm{M}$ solution of isobutyraldeyhyde $(0.27 \mathrm{mmol})$ in toluene was added to the solution, and the reaction was allowed to stir for $12 \mathrm{~h}$. The cap was removed from the reaction and $60 \mu \mathrm{L}(0.54 \mathrm{mmol})$ iodobenzene was added followed by $0.90 \mathrm{~mL} 3 \mathrm{M}$ aqueous $\mathrm{KOH}$. The reaction was heated to $95{ }^{\circ} \mathrm{C}$ for 6 hours. It was allowed to cool to room temperature and was quenched with saturated aqueous $\mathrm{NH}_{4} \mathrm{Cl}$. The mixture was extracted with ethyl acetate $(2 \times 5 \mathrm{~mL})$. The organic layers were dried over anhydrous $\mathrm{Na}_{2} \mathrm{SO}_{4}$, filtered, and the solvent removed by rotary evaporation. The crude material was purified by silica gel chromatography (10:1 hexanes:ethyl acetate) to provide $53 \mathrm{mg}$ (74\%) of 2-methyl-5,6-diphenylhex-5-en3 -ol as a clear yellow oil.

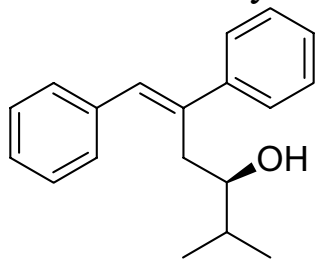

$(\boldsymbol{R})$-2-Methyl-5,6-diphenylhex-5-en-3-ol (8). Clear yellow oil. $\mathrm{R}_{\mathrm{f}}=0.47$ in 5:1 hexanes/ethyl acetate. IR $\left(\mathrm{CH}_{2} \mathrm{Cl}_{2}\right) 2597(\mathrm{~m}), 3056(\mathrm{~s}), 2964(\mathrm{~m}), 1600(\mathrm{~m}), 1495$ (m), $1447(\mathrm{~m}) \mathrm{cm}^{-1} ;{ }^{1} \mathrm{H}$ NMR $\left(400 \mathrm{MHz}, \mathrm{CDCl}_{3}\right): \delta 7.51-7.49(4 \mathrm{H}, \mathrm{m}), 7.43-7.26$ $(6 \mathrm{H}, \mathrm{m}), 6.92(1 \mathrm{H}, \mathrm{s}), 3.52-3.47(1 \mathrm{H}, \mathrm{m}), 3.04(1 \mathrm{H}, \mathrm{dd}, J=14.4,10.0 \mathrm{~Hz}), 2.85$ $(1 \mathrm{H}, \mathrm{dd}, J=14.4,2.8 \mathrm{~Hz}), 1.76-1.71(1 \mathrm{H}, \mathrm{m}), 0.93(6 \mathrm{H}, \mathrm{dd}, J=7.4 \mathrm{~Hz}),{ }^{13} \mathrm{C}$ NMR $\left(100 \mathrm{MHz}, \mathrm{CDCl}_{3}\right): \delta 142.6,139.9,137.7,131.6,129.1,128.6,128.3,127.5,126.9$, 126.7, 74.5, 34.6, 33.6, 18.7, 17.5; LRMS (ESI) Calc'd for $\mathrm{C}_{19} \mathrm{H}_{22} \mathrm{O}(\mathrm{M}+\mathrm{Na})^{+}$: 289.4 Found $(\mathrm{M}+\mathrm{Na})^{+}$: 289.2 .

The $\%$ ee of the product was determined by ${ }^{1} \mathrm{H}$ NMR analysis of the Mosher ester.
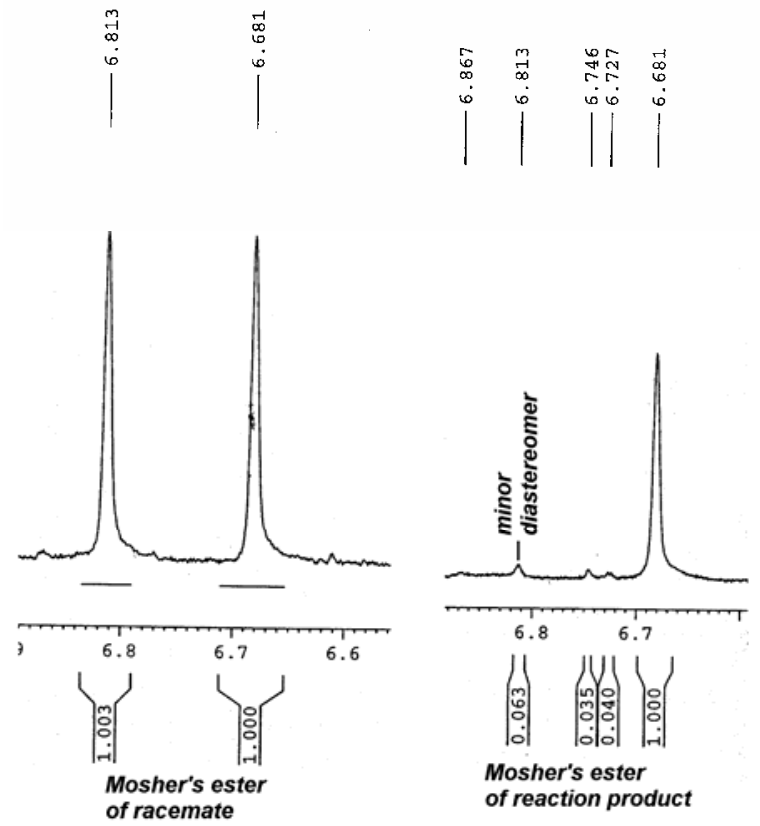

Page S- 15 


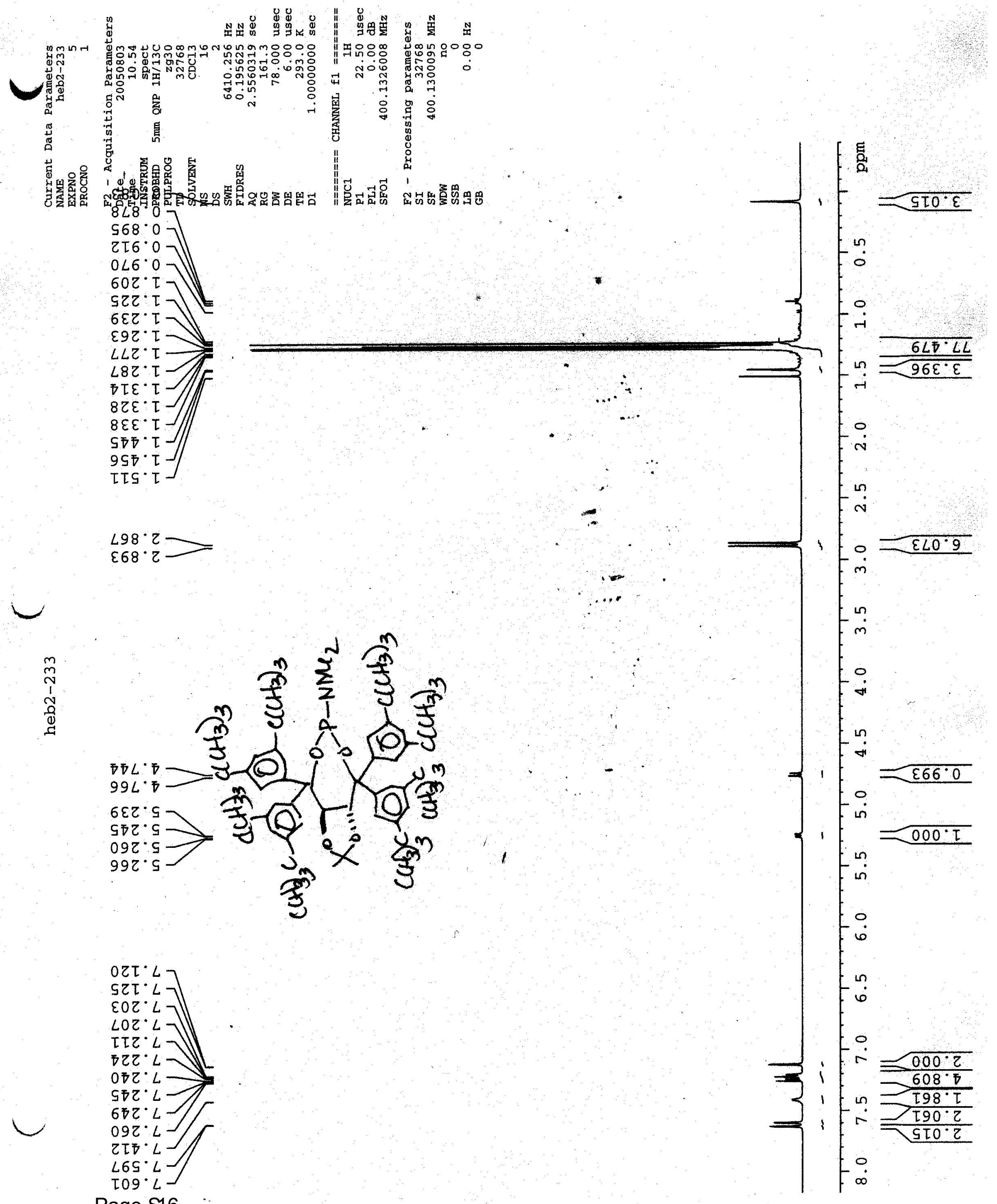




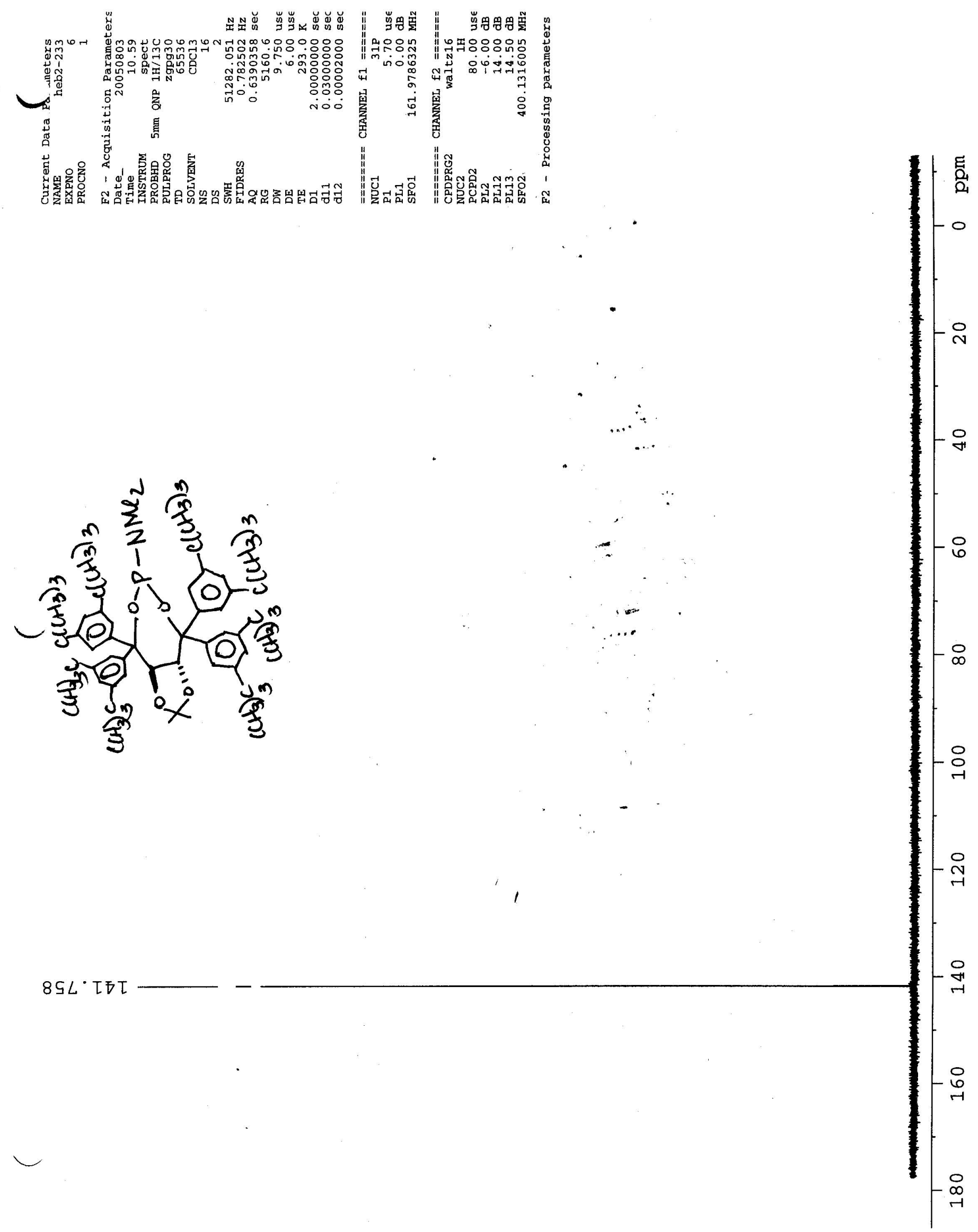

Page $\$ 7$ 

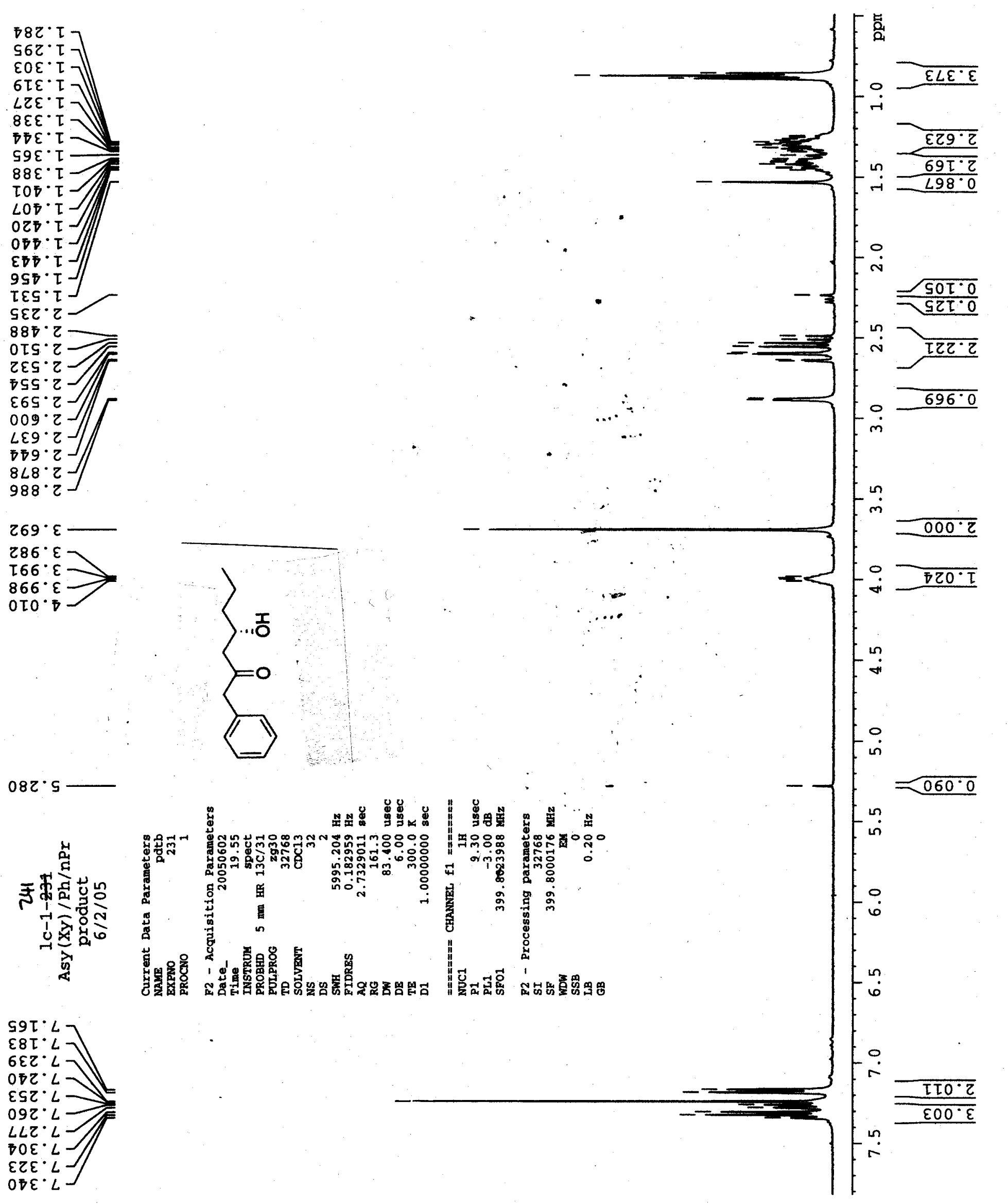

$082 \cdot 5$
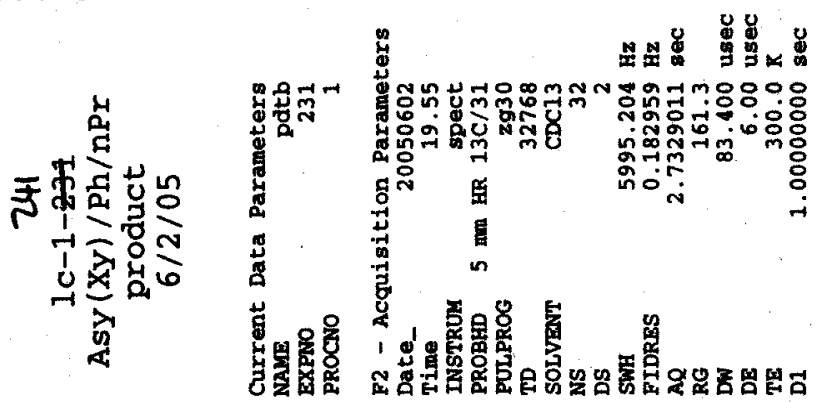

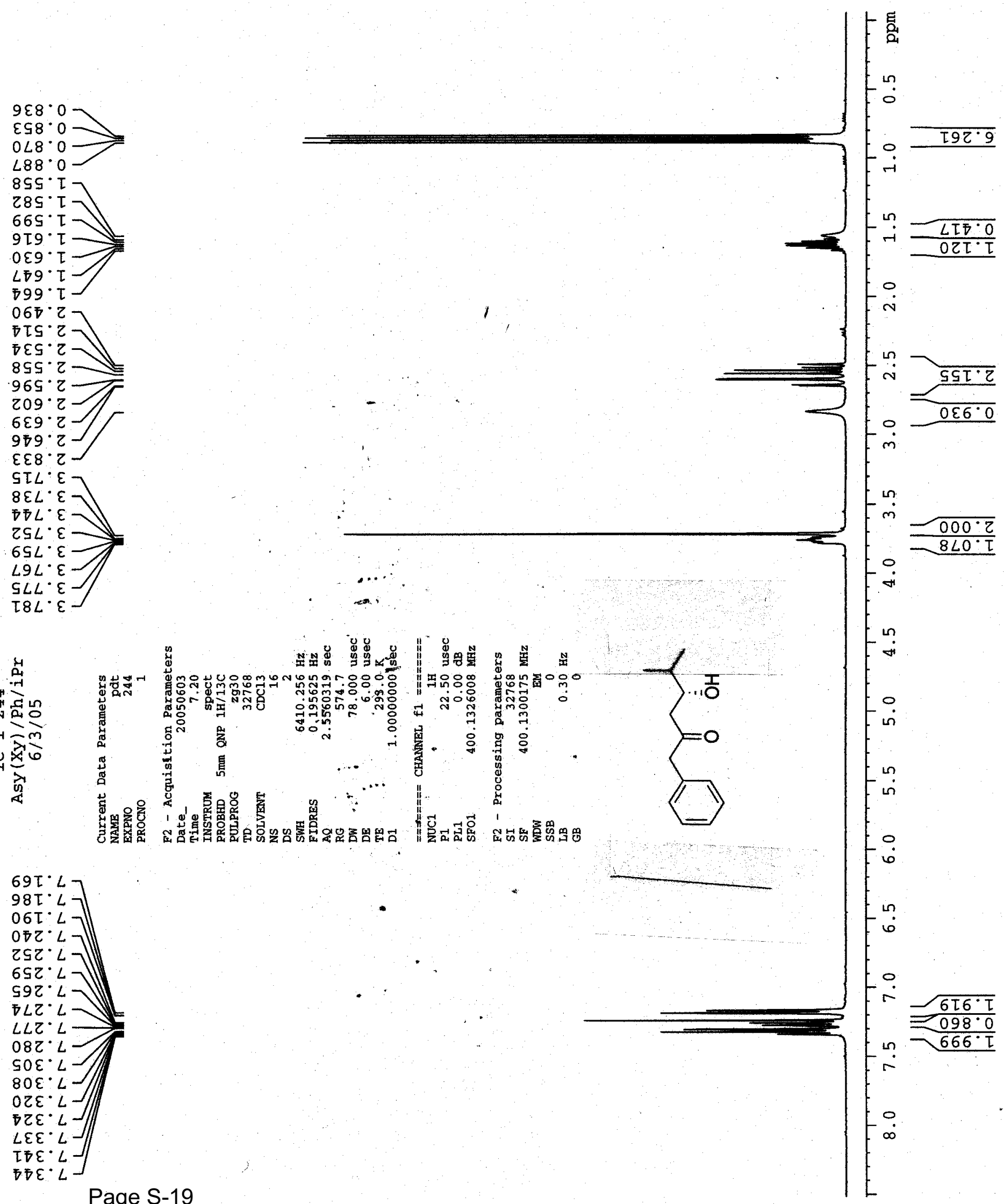

Page S-19 


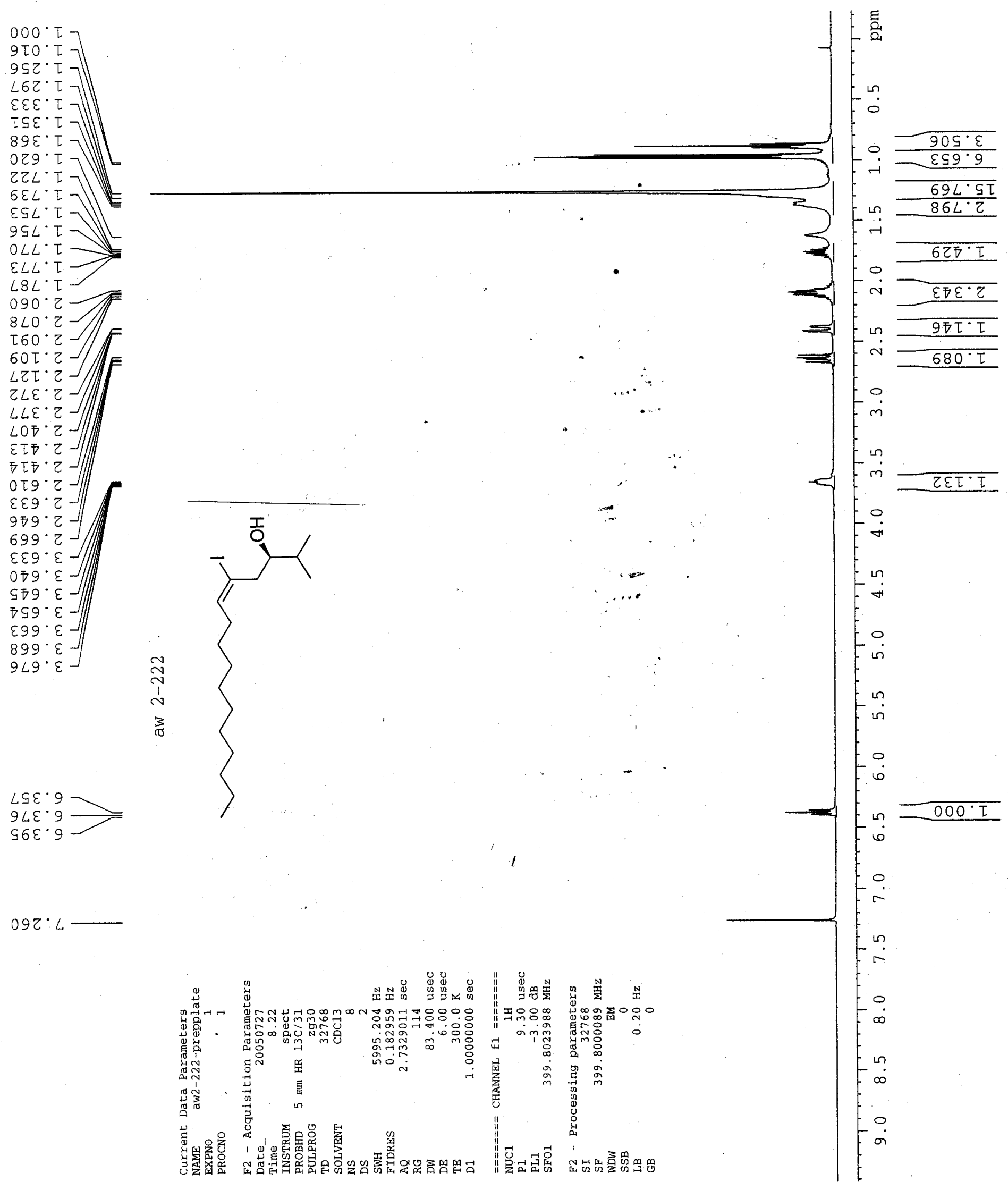




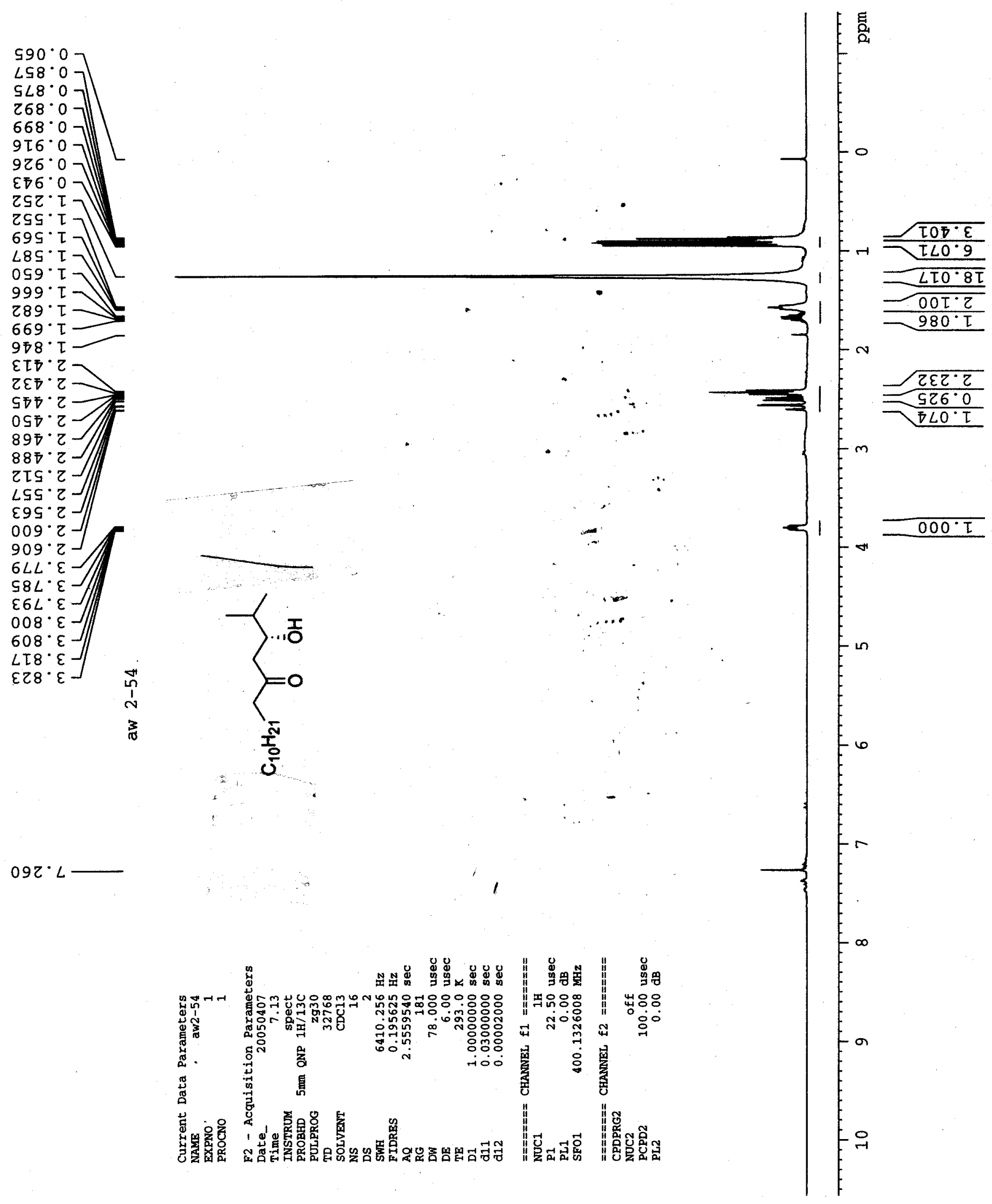


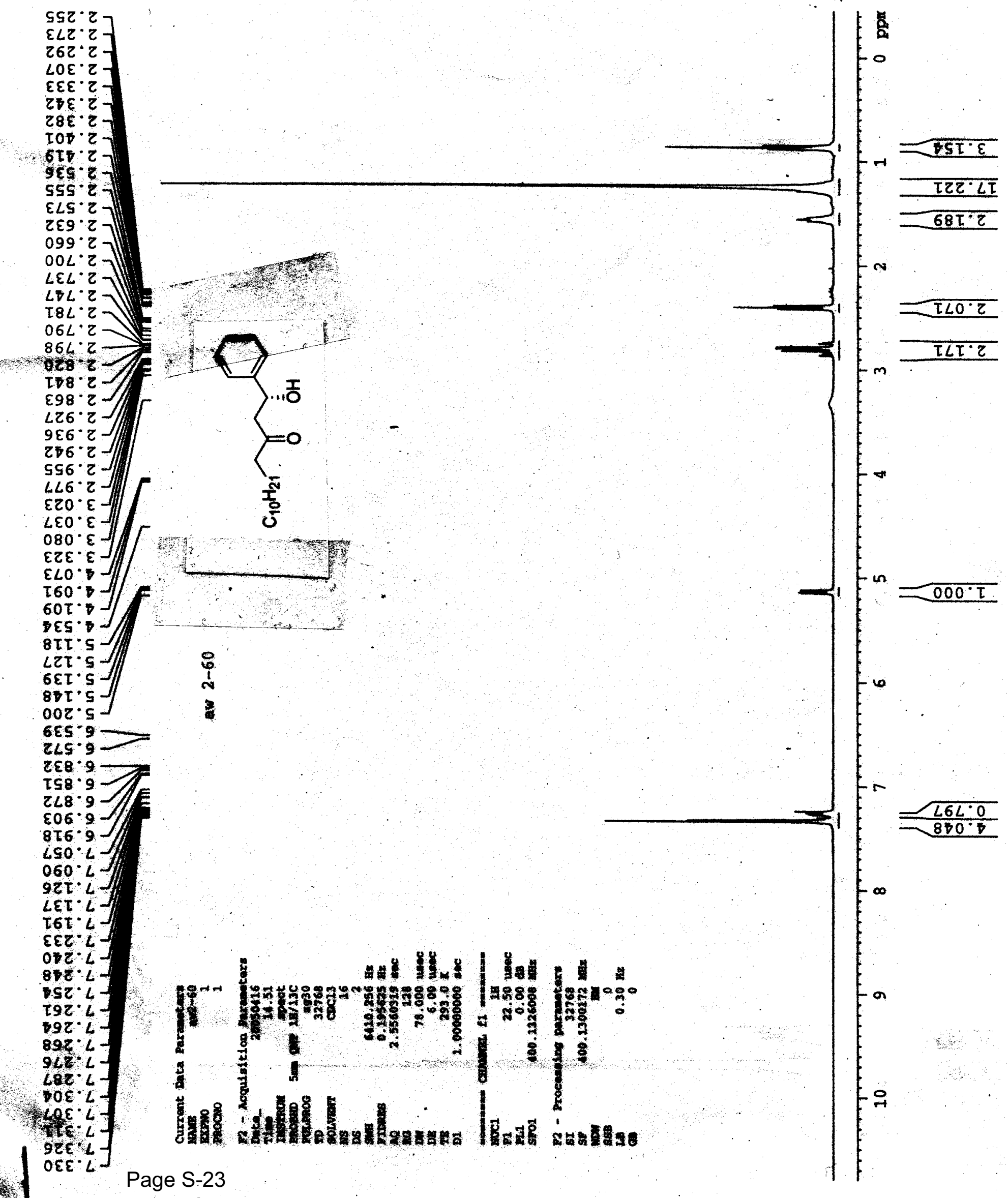



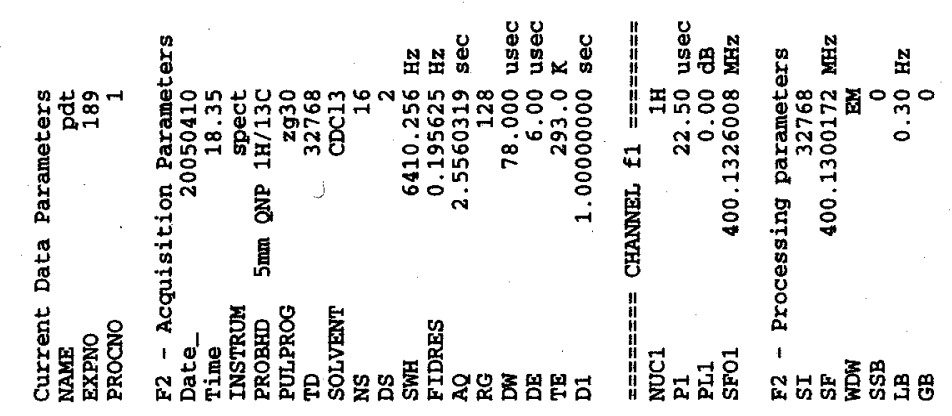

运
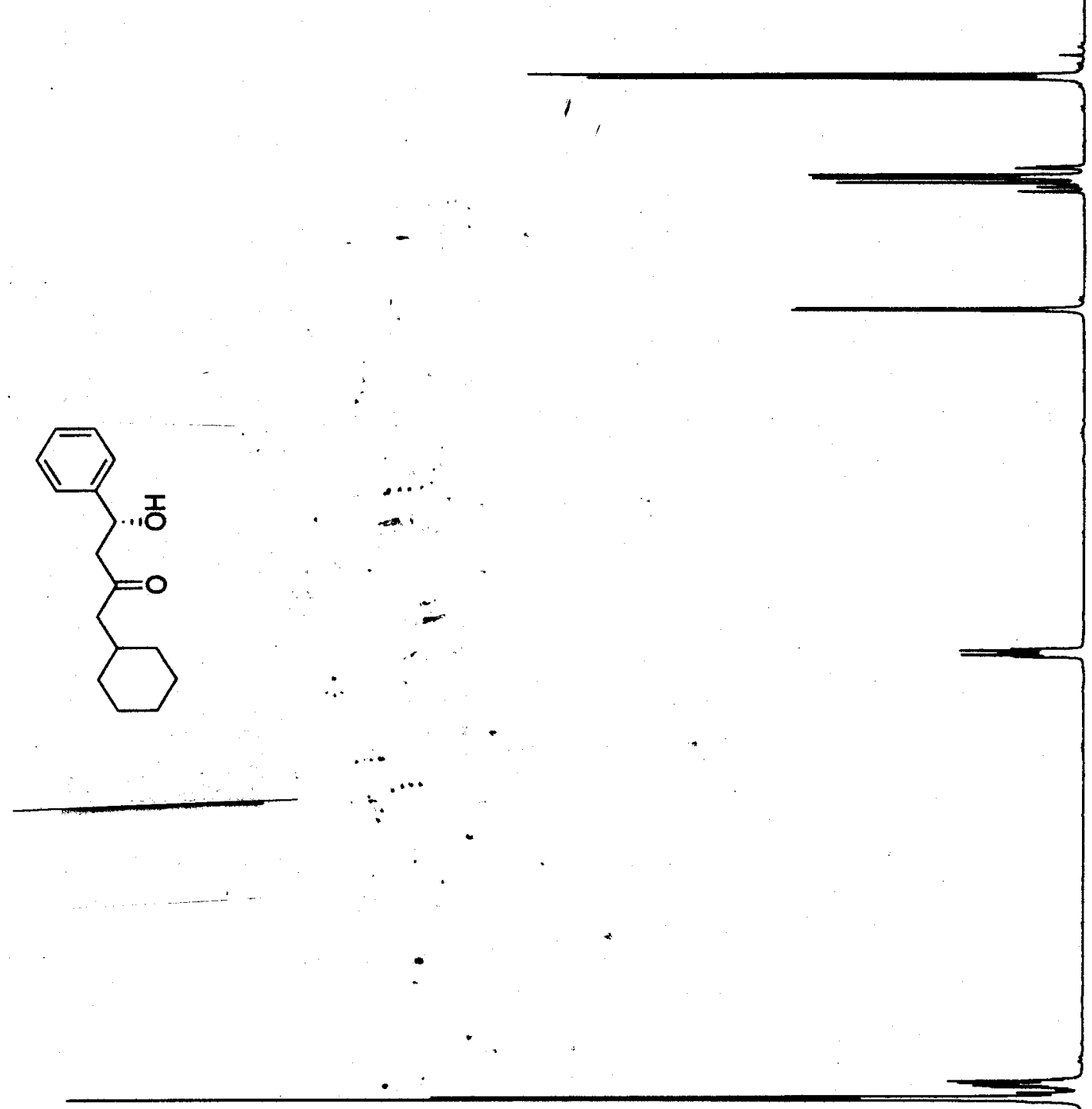

$-80 \tau \cdot \tau$

in

0II.

$m$

n? $\lcm{296^{\circ} 0}$

$m$

$-0$

$-\stackrel{4}{*}$

$+$

-

is $000^{\circ} \mathrm{I}$

- in

เ

0

-

ㅇ.

$r$

$\frac{6 T^{\circ} \tau}{096^{\circ} \varepsilon}$

r

-

$\infty$

$\stackrel{\infty}{\infty}$ 


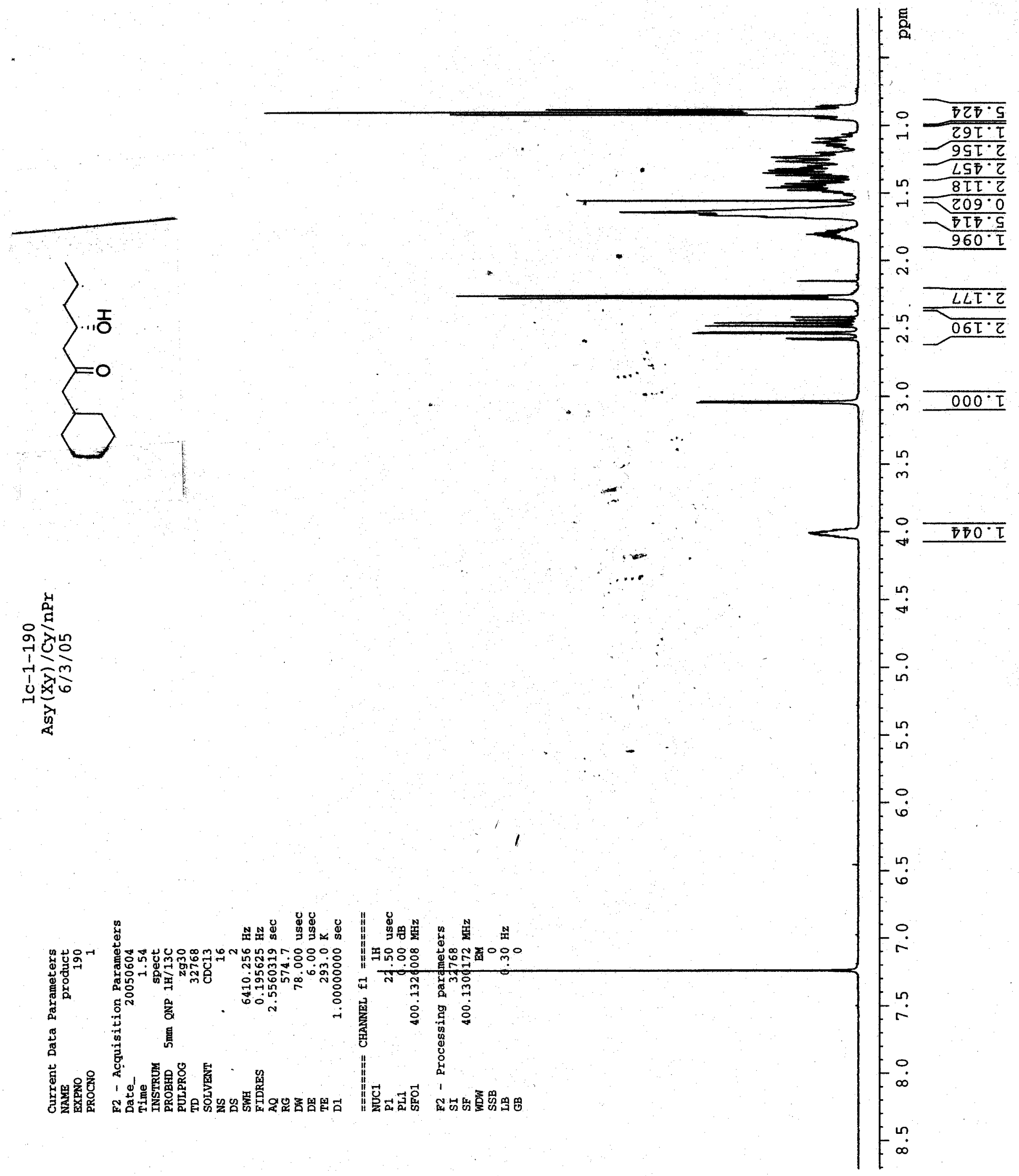

Page S-25 

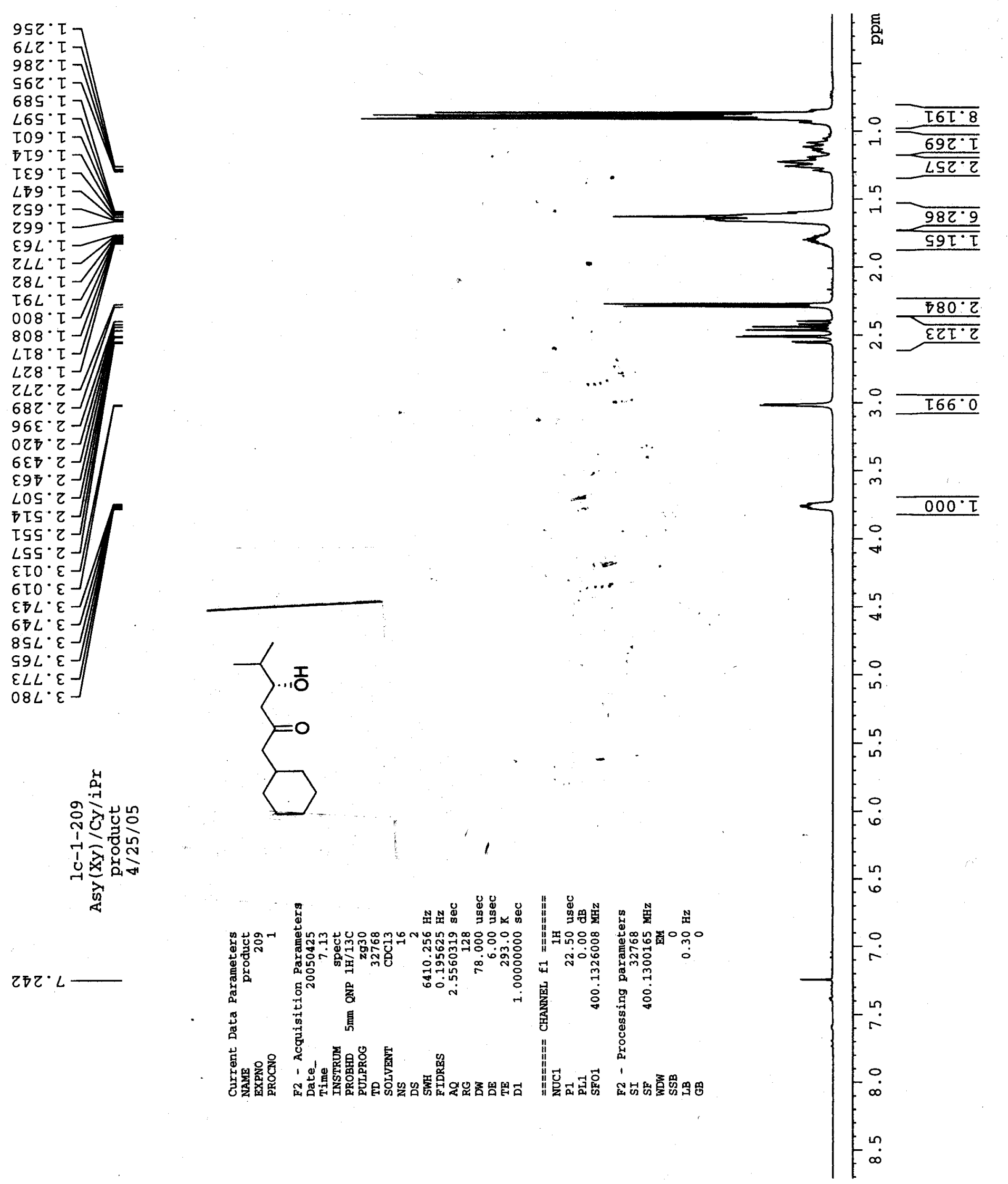


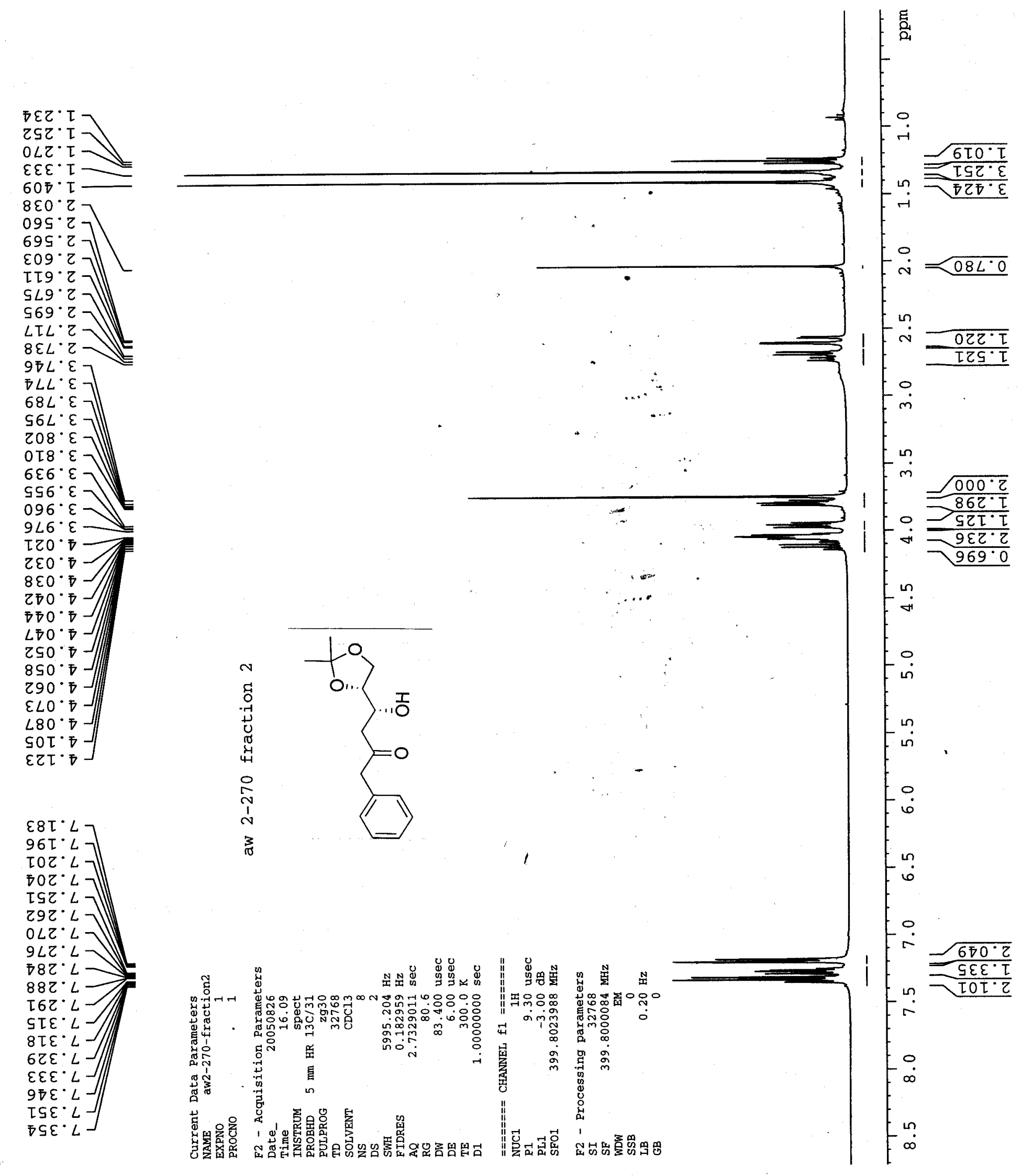




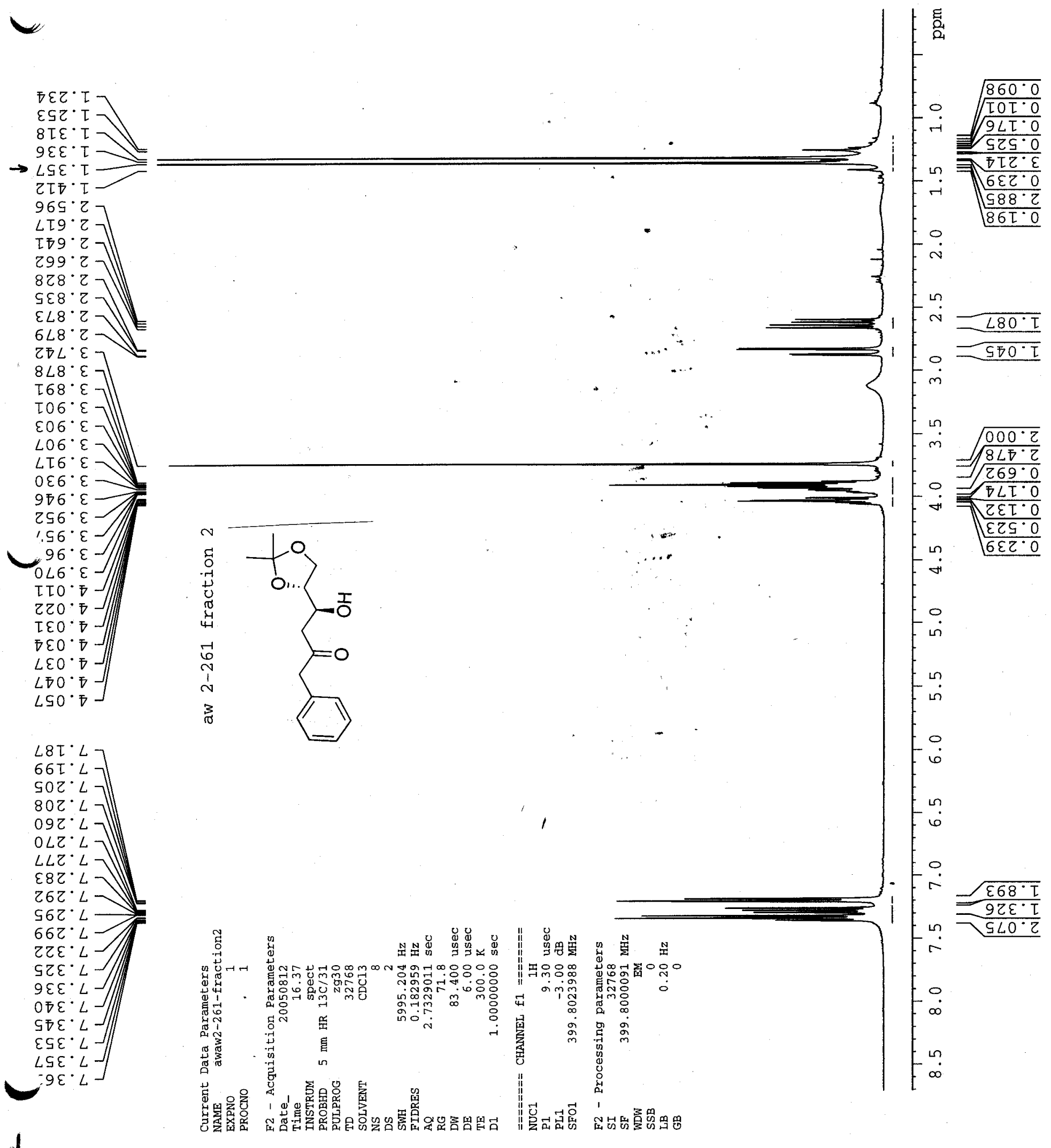



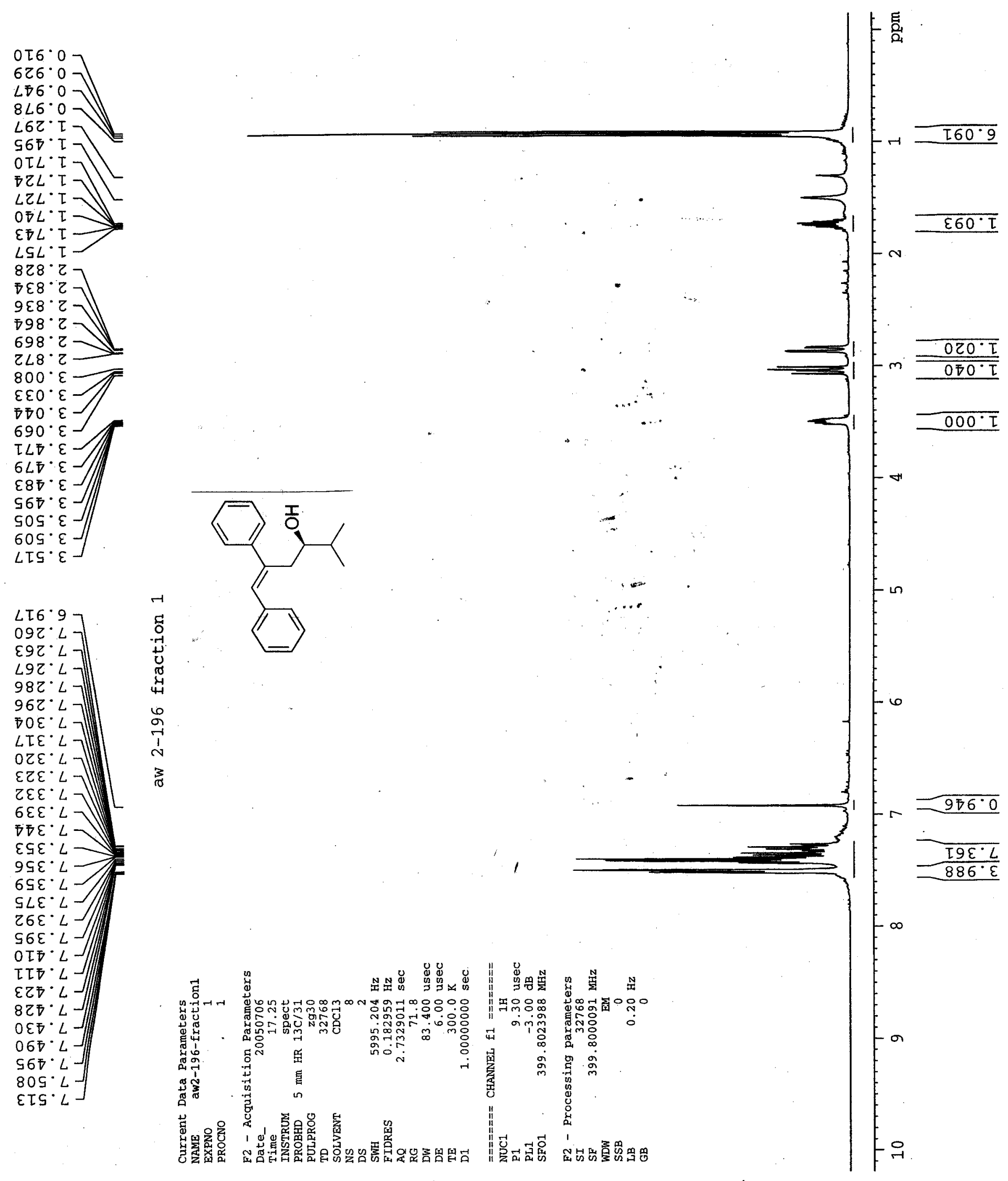

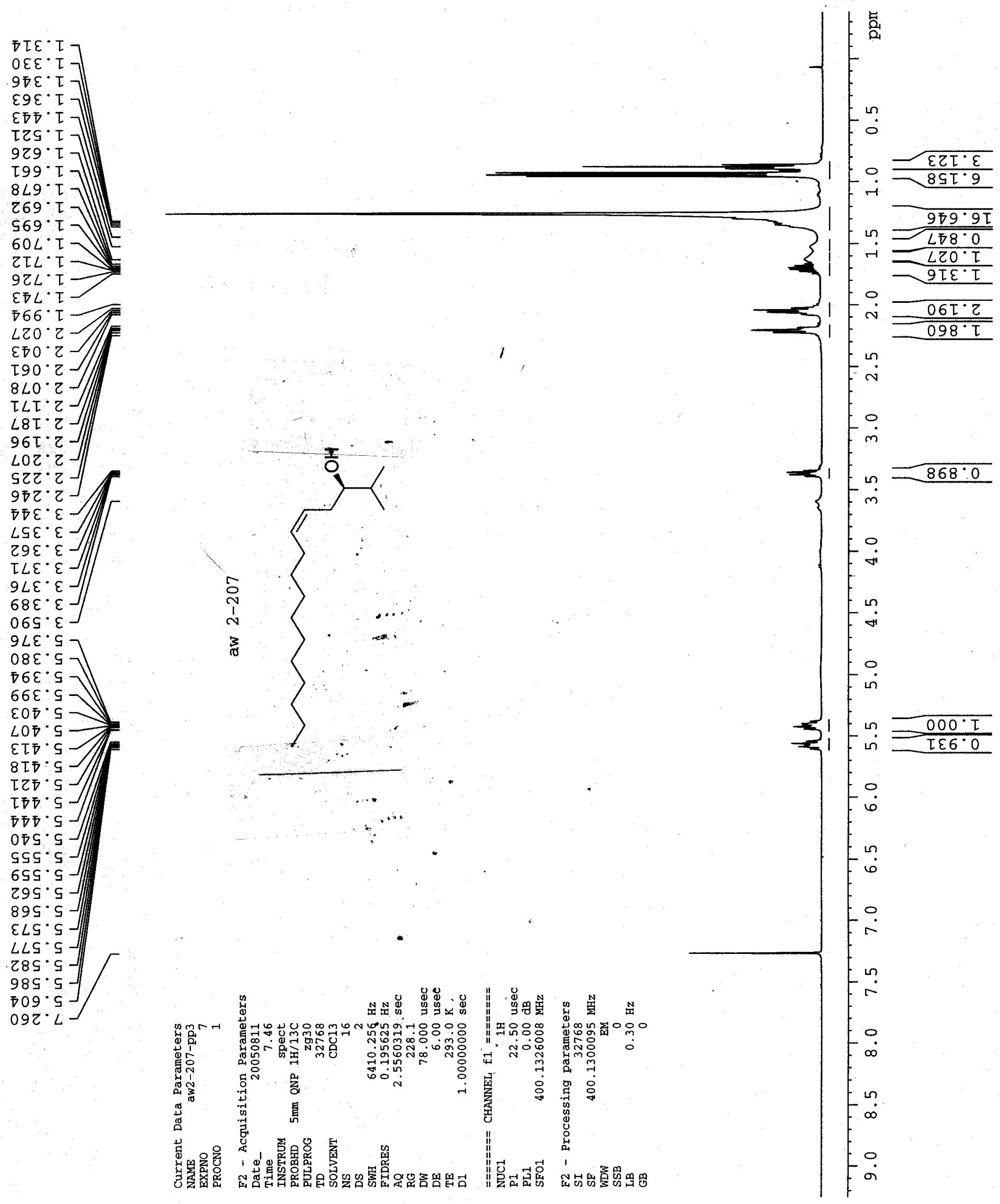